\title{
A methodology for in silico endovascular repair of abdominal aortic aneurysms
}

\author{
André Hemmler • Brigitta Lutz • Christian Reeps · Günay Kalender • \\ Michael W. Gee
}

Received: date / Accepted: date

\begin{abstract}
Endovascular aneurysm repair (EVAR) can involve some unfavorable complications such as endoleaks or stent-graft (SG) migration. Such complications, resulting from the complex mechanical interaction of vascular tissue, SG and blood flow or incompatibility of SG design and vessel geometry, are difficult to predict. Computational vascular mechanics models can be a predictive tool for the selection, sizing and placement process of SGs depending on the patient-specific vessel geometry and hence reduce the risk of potential complications after EVAR. In this contribution, we present a new in silico EVAR methodology to predict the final state of the deployed SG after intervention and evaluate the mechanical state of vessel and SG, such as contact forces and wall stresses. A novel method to account for residual strains and stresses in SGs, resulting from the precompression of stents during the assembly process of SGs, is presented. We suggest a parameter continuation approach to model various different sizes of SGs within one in silico EVAR simulation which can be a valuable tool when investigating the issue of $\mathrm{SG}$ oversizing. The applicability and robustness of the pro-
\end{abstract}

André Hemmler · Michael W. Gee (凶)

Mechanics \& High Performance Computing Group, Technische Universität München, Parkring 35, 85748 Garching b. München, Germany

E-mail: gee@tum.de

Brigitta Lutz · Christian Reeps

Universitätsklinikum Carl Gustav Carus Dresden, Klinik für Viszeral-, Thorax- und Gefäßchirurgie, Fetscherstraße 74, 01307 Dresden, Germany

Günay Kalender

DRK Kliniken Berlin, Klinik für vaskuläre und endovaskuläre Chirurgie, Salvador-Allende-Straße 2-8, 12559 Berlin, Germany posed methods are demonstrated on the example of a synthetic abdominal aortic aneurysm geometry.

Keywords abdominal aortic aneurysm · endovascular aneurysm repair · stent-graft · oversizing · finite element method

\section{Introduction}

An abdominal aortic aneurysm (AAA) is characterized by an abnormal dilatation of the infrarenal aorta. In order to assess AAA rupture risk the aneurysm diameter and the rate of growth [62] are used. Once the risk of rupture of an AAA outbalances the risk of surgery, the surgeon has to decide between a conventional open repair and an endovascular aneurysm repair (EVAR) where a stent-graft (SG) is deployed inside the AAA to exclude the aneurysm sac from the main blood flow. Most SGs are composed of a wire mesh (stent) which is sewn on a polymeric fabric (graft). In order to exclude the AAA both ends of the SG need to be attached in a "healthy" part of the vessel, i.e. a part of the aorta without dilatation. The part where SG and "healthy" vessel overlap is called landing zone or sealing zone. The landing zone requires certain conditions, such as a certain length, to properly seal the aneurysm leakproof. Although EVAR is less invasive for candidates and is associated with reduced 30-day operative mortality rate [33], it is not free of complications and might require secondary interventions. The most frequent complications after EVAR are (i) endoleaks associated with an inappropriate seal between SG and vessel wall and ongoing aneurysm rupture risk [34,12], (ii) SG migration $[75,74,2]$, (iii) neck dilatation resulting from SG induced tissue-overstress and tissue remodeling [48,74], 
(iv) graft kinking resulting in occlusion of blood vessels $[14,55]$, and (v) SG fracture [46,5]. The mechanical behavior of the SG, the biomechanical behavior and physiological state of the vessel appear to have an essential impact on the occurrence of these complications $[83,12,2,42]$.

The objective of this work is to develop an in silico EVAR methodology to predict the final state of the deployed SG after intervention. We aim to find the final SG position in the vessel geometry under static conditions rather than reproducing the intra-operative steps of EVAR. The methodology has to be applicable to any patient-specific AAA and any type of SG. The results of the final state of $\mathrm{SG}$ and vessel shall be evaluated with respect to the mechanical state of vessel and SG, such as wall stresses as well as contact forces between SG and vessel. Throughout this work, we use the definitions given in table 1 as the notation in literature is not unique.

Table 1 Definition of terms.

\begin{tabular}{l}
\hline \hline$S G$ placement: \\
Process of positioning the SG within the vessel. \\
SG deployment: \\
Process of removing the delivery system and therefore \\
allowing the SG to freely deform within the vessel. \\
Stent predeformation: \\
Stents generally are manufactured with a diameter \\
larger than the associated graft. Stents are radially \\
compressed during the assembling process of SGs \\
resulting in an assembled SG with residual strains and \\
stresses. The modeling approach to consider this effect \\
is denoted as stent predeformation. \\
Vessel prestress: \\
Geometry reconstruction from medical imaging such as \\
CT scans involve an initial geometric configuration \\
that is not stress-free. Vessel prestressing is the \\
modeling approach of initializing the vessel model to \\
this stressed configuration. \\
Landing zone: \\
The most proximal/distal part of the SG which is \\
directly attached to the luminal vessel surface and \\
which is responsible for proper sealing between SG and \\
vessel. \\
SG oversize: \\
luminal vessel diameter in the landing zone of the SG. \\
\hline \hline
\end{tabular}

Computational vascular mechanics models can be a promising tool to get deeper insight into the mechanical state of vessel and SG after the insertion of the SG which cannot be received from medical imaging. The additional information of the mechanical state of vessel and SG can be helpful in further localizing the source of SG related complications and help to give answers to open question such as the best value for SG oversizing [75,9]. Applied to clinical cases, an in silico
EVAR methodology can be used as predictive tool for a better risk assessment of the intervention indicating potential SG related complications already in the preoperative planning phase. SG selection and sizing is one of the most difficult steps in the pre-operative planning phase as this step requires a lot of experience of the surgeon and strongly depends on the patient-specific case $[26,43]$. Some oversizing of the SG with respect to the luminal vessel diameter is necessary to obtain an adequate seal and fixation of the $\mathrm{SG}$ in the proximal and distal landing zones $[75,9,74]$. But excessive SG oversizing might lead to negative results as well. Most frequently stated EVAR complications arising from excessive SG oversizing are (i) tissue-overstress [48,74] related to growth and remodeling of the tissue, vessel dilatation and subsequent SG migration or endoleaks, and (ii) incomplete SG expansion [45,52,41]. The instructions for use of marketed SGs generally suggest an oversizing of $10-20 \%$, however, there is no consensus with regard to optimal degree of SG oversizing and even SG oversizing above $30 \%$ is practically used $[75,74,56]$. A computational, predictive tool can be very valuable in finding the optimal SG type and size which depends on the patient-specific vessel geometry [83]. Hence, it further reduces the risk of EVAR related complications and the risk of secondary treatments.

The process of SG placement and deployment is a highly nonlinear process mainly due to the occurrence of complex contact scenarios between SG and vessel, buckling of the very thin graft as well as nonlinear material behaviors. Hence, numerical modeling of this process is challenging and the methodology how the SG is placed and deployed within the vessel are essential for the efficiency and robustness of the approach. Stent predeformation [72] as well as in vivo non-stress-free vessel geometries [30] require special computational techniques which have to be consistently integrated into the mechanical framework. High complexity of vessel and SG shapes and their large inter-patient variability are further challenges of the presented in silico EVAR methodology.

The presented methods are based on finite element methods (FEM) and a novel 3D morphing algorithm for SG geometries which is closely related to morphing algorithms originally used in image processing (e.g. Beier et al. [6], Lerios et al. [51] or Lazarus et al. [50]) and sweeping algorithms (e.g. Wang et al. [81]). In this methodology, the intra-operative steps of EVAR are strongly simplified to reduce the numerical complexity of the problem. This means, e. g. medical devices such as guidewires are not considered in the in silico EVAR approach. 
Mortar based frictional contact [68] is considered between the vessel and the SG model which both can undergo finite deformations. Further, attention is payed to detailed modeling of the vessel. This includes an anisotropic and hyperelastic law for the vessel wall as well as the consideration of the constituents intraluminal thrombus (ILT) and calcifications since these constituents are known to be able to have a distinct impact on the success of the EVAR treatment $[83,73,82]$. Furthermore, we apply a blend between vessel material models for a "healthy" vessel wall and an "aneurysmatic" vessel wall as both have been shown to exhibit distinct material behavior $[61,77]$. This differentiation between "healthy" and "aneurysmatic" vessel wall is quite essential as in general the landing zone of the SG is in the region of "healthy" vessel tissue and not in the diseased aneurysmatic part [34].

Stent predeformation should be considered in an in silico EVAR simulation to be able to compute contact forces between SG and vessel more accurately [72]. We propose a stent predeformation methodology which is based on the alteration of the stress-free reference configuration of the stent after SG assembly. This approach is flexible and does not require any additional assembling simulation or mechanical experiments for parameter fitting. Instead, it can be implicitly integrated within the actual in silico EVAR methodology.

Addressing the question of SG sizing, we suggest a parameter continuation approach. It allows to model various different diameters of SGs within one in silico EVAR simulation and thus results in an enormous saving in simulation time compared to other approaches $[69,16]$. Furthermore, the parameter continuation approach allows for a continuous variation of the SG diameter which is not possible when doing separate simulations for distinct SG choices.

Several studies have already been conducted in the field of vascular modeling and in silico EVAR simulations. Among others, Humphrey and Holzapfel [40], Gasser et al. [27], Maier et al. [54], Vande Geest et al. [76] and Fillinger et al. [25] focused on computational models of AAAs mainly to assess the rupture risk. Kleinstreuer et al. [46], De Bock et al. [15], Demanget et al. [20,21] and Roy et al. [72] performed experimental and numerical tests on marketed SGs considering the mechanical behavior of stent and graft.

Other groups (e.g. Holzapfel et al. [39], Auricchio et al. [3], Mortier et al. [60], Iannaccone et al. [41,17] and Morlacchi et al. [59]) studied the virtual deployment of stents which can be seen as pioneering works for in silico EVAR simulations. Some in silico EVAR simulations of SGs in idealized aneurysms or vessels (e.g. De Bock et al. $[16,18]$ and Perrin et al. [66]) and some in patient- specific vessels (Auricchio et al. [4], Perrin et al. [64, 65]) exist.

In most previous in silico EVAR studies [4,16,18, 66], the in silico EVAR methodology was adopted from virtual stent deployment simulations. The methodology consists of a rigid, cylindrical catheter in which the SG is radially crimped, placed within the vessel and finally deployed within the vessel. Perrin et al. [64, 65] presented a different in silico EVAR methodology which was applied to patient-specific and bifurcated AAA geometries. In this methodology, first the SG was crimped inside a rigid, tubular catheter and afterwards the catheter was deformed onto the pre-operative geometry of the patients vessel. Contact constraints forced the SG to remain inside the catheter and gradually deformed the SG. Finally, vesel material properties were assigned to the catheter allowing the catheter to deform elastically.

A major challenge of all previously mentioned in silico EVAR methodologies was the high complexity of the problem arising from the high degree of nonlinearity mainly due to buckling of the graft and complex contact phenomena. The highest degree of complexity occurs during crimping and placement as in these configurations the $\mathrm{SG}$ is radially compressed and the graft strongly buckles. The only published in silico EVAR methodology that avoids the high degree of complexity during crimping and placement of the SG is presented by Prasad et al. [69]. They inflated the vessel by an unphysiological pressure increase to make room for the SG and subsequently released this artificial pressure during the virtual deployment step. This approach however is restricted to simple vessel geometries. The objective of all previously mentioned studies of in silico EVAR was to obtain the deployed configuration of the SG inside the vessel rather than reproducing the mechanically and numerically complex single steps of an EVAR intervention. Hence, there actually is no need for representing this high degree of complexity during crimping and placement of the SG as long as the influence of these steps on the deployed configuration is small. This raises the interest for an in silico EVAR methodology which avoids extremely complex steps during placement of the SG but nevertheless provides a high level of accuracy of the deployed SG in the patient-specific vessel.

Although numerous studies on the mechanical behavior of SGs had been performed, the stent predeformation was rarely considered. Perrin et al. [65] developed a mechanically sophisticated SG assembling strategy for Anaconda ${ }^{\text {TM }}$ SGs (Vascutek, Inchinnan,UK) which considered the predeformed stent configuration. Roy et al. [72], who called this effect "stent pre-load", developed a more general rule for Cook Zenith ${ }^{\circledR}$ SGs 
(Cook Medical, Bloomington, Indiana, USA). They augmented the Young's modulus of the stent by a factor of 2 to consider the effect of stent predeformation. This was based on the assumption of a linear relationship between radial force and diameter reduction of stents and required mechanical tests for calibration.

Some studies (e.g. Prasad et al. [69] or De Bock et al. [16]) numerically investigated the issue of SG oversizing after having applied one of the aforementioned in silico EVAR methodologies. For each degree of SG oversizing that was considered a separate in silico EVAR simulation was applied. Consequently, investigating different ranges of oversizing was computationally expensive and only discrete realizations of oversizing could be investigated.

The outline of this paper is as follows: in section 2 we introduce the mathematical description of the 3D morphing algorithm and the in silico EVAR methodology as well as constitutive models of SG and vessel. In section 3, a tube demonstrator is used to perform convergence studies of the $3 \mathrm{D}$ morphing algorithm and to show its robustness. Also, the in silico EVAR methodology is applied to a synthetic AAA geometry. Using this geometry, a continuous variation of the degree of SG oversize is investigated by application of the parameter continuation approach. The influence of the degree of SG oversize on SG and vessel is analysed. Further, we compare the results obtained by the parameter continuation approach to a "direct" in silico EVAR simulation to be able to quantify the error induced by the parameter continuation approach. At the end of section 3, we examine the influence of stent predeformation on internal stress states of stent and graft as well as on contact tractions and vessel stresses in the deployed state of the SG. The findings of section 3 are discussed in section 4 . Limitations and conclusions are drawn in section 5 and 6 , respectively.

\section{Materials and methods}

\subsection{Model assumptions}

We use a model which incorporates the following basic assumptions:

- No medical tools, such as e.g. guidewires, other than the SG itself are considered.

- The simplified intra-operative steps only have a minor influence on the final deployed SG configuration.

- Friction between SG and vessel is modeled as dry friction using Coulomb's law. Lubrication is neglected.

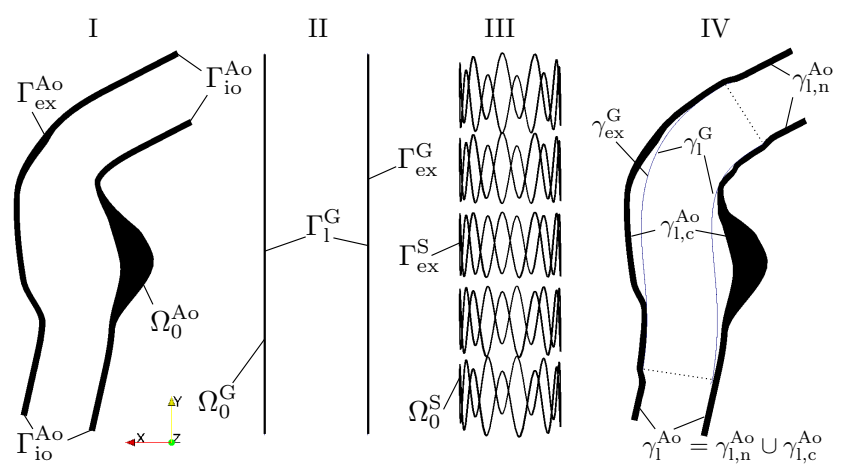

Fig. 1 Domains and boundaries of the abdominal aorta $\Omega_{0}^{\text {Ao }}$ (I), the graft $\Omega_{0}^{\mathrm{G}}$ (II) and the stent $\Omega_{0}^{\mathrm{S}}$ (III) in the reference configuration and in the current configuration (IV). $(\bullet)_{\text {ex }}$ denotes the exterior boundaries of the aorta $\Gamma_{\mathrm{ex}}^{\mathrm{Ao}}$, the graft $\Gamma_{\mathrm{ex}}^{\mathrm{G}}$ and the stent $\Gamma_{\mathrm{ex}}^{\mathrm{S}} ;(\bullet)_{1}$ describes the luminal boundaries of the aorta covered by the graft $\gamma_{1, c}^{\text {Ao }}$ and not covered by the graft $\gamma_{1, \mathrm{n}}^{\mathrm{Ao}}$ as well as the luminal boundary of the graft $\Gamma_{1}^{\mathrm{G}}$; $\Gamma_{\text {io }}^{\text {Ao }}$ denotes the boundary at the in- and outlet of the aortic segment.

- Treatment as purely solid mechanics problem. For now, fluid dynamics of the blood flow is neglected. A quasi-static pressure state is considered.

- Variability in patient-specific vessel material properties is neglected. Population averaged mean values are used for vessel material parameters.

- Constant vessel wall thickness is assumed.

\subsection{D nonlinear elastostatics including frictional contact}

We consider the boundary value problem (BVP) of multibody finite deformation elastostatics with frictional contact:

$$
\begin{array}{lc}
\nabla \cdot\left(\boldsymbol{F}^{(\Pi)} \boldsymbol{S}^{(\Pi)}\right)+\boldsymbol{b}_{0}=\mathbf{0} & \text { in } \Omega_{0}^{(\Pi)}, \\
\boldsymbol{\sigma}^{(\Pi)} \boldsymbol{n}^{(\Pi)}=\hat{\boldsymbol{t}}^{(\Pi)} & \text { on } \gamma_{\sigma}^{(\Pi)}, \\
\left(\boldsymbol{F}^{\mathrm{Ao}} \boldsymbol{S}^{\mathrm{Ao}}\right) \boldsymbol{N}^{\mathrm{Ao}}=-\hat{k}_{\mathrm{ex}} \boldsymbol{u}^{\mathrm{Ao}} & \text { on } \Gamma_{\mathrm{ex}}^{\mathrm{Ao}}, \\
\left(\boldsymbol{F}^{\mathrm{Ao}} \boldsymbol{S}^{\mathrm{Ao}}\right) \boldsymbol{N}^{\mathrm{Ao}}=-\hat{k}_{\mathrm{io}} \boldsymbol{u}^{\mathrm{Ao}} & \text { on } \Gamma_{\mathrm{io}}^{\mathrm{Ao}}, \\
\boldsymbol{u}^{\mathrm{S}}=\boldsymbol{u}^{\mathrm{G}} & \text { on } \Gamma_{\mathrm{m}}, \\
g_{\mathrm{n}} \geq 0, \quad\left\|\boldsymbol{t}_{\mathrm{n}}\right\| \leq 0, \quad\left\|\boldsymbol{t}_{\mathrm{n}}\right\| g_{\mathrm{n}}=0 & \text { on } \gamma_{\mathrm{ex}}^{\mathrm{G}}, \\
\Lambda:=\left\|\boldsymbol{t}_{\tau}\right\|-\mu\left\|\boldsymbol{t}_{\mathrm{n}}\right\| \leq 0, & \\
\boldsymbol{v}_{\tau, \mathrm{rel}}+\beta \boldsymbol{t}_{\tau}=\mathbf{0}, & \\
\beta \geq 0, \quad \Lambda \beta=0 & \text { on } \gamma_{\mathrm{ex}}^{\mathrm{G}},
\end{array}
$$

where $\Omega_{0}^{(\Pi)}$ with $\Pi=\{\mathrm{Ao}, \mathrm{G}, \mathrm{S}\}$ is the reference configuration of body $\Pi$. "Ao" denotes the vessel, "S" the stent and "G" the graft (Figure 1). The displacements $\boldsymbol{u}^{(\Pi)}$, being the state variables, relate the coordinates of the reference configuration 
$\boldsymbol{X}^{(I)} \in \Omega_{0}^{(\Pi)}$ with the coordinates of the current configuration $\boldsymbol{x}^{(\Pi)} \in \Omega^{(\Pi)}$ by the diffeomorphic mapping

$\boldsymbol{\Phi}\left(\boldsymbol{X}^{(\Pi)}\right)=\boldsymbol{x}^{(\Pi)}=\boldsymbol{X}^{(\Pi)}+\boldsymbol{u}^{(\Pi)}$.

Further, $\boldsymbol{F}^{(\Pi)}=\mathbf{1}+\nabla \boldsymbol{u}^{(\Pi)}$ is the deformation gradient, $\boldsymbol{S}^{(I)}$ the second Piola-Kirchhoff stress tensor and $\boldsymbol{\sigma}^{(I)}=\frac{1}{\operatorname{det}\left(\boldsymbol{F}^{(I)}\right)} \boldsymbol{F}^{(\Pi)} \boldsymbol{S}^{(\Pi)}\left(\boldsymbol{F}^{(\Pi)}\right)^{\mathrm{T}}$ the Cauchy stress tensor. $\hat{\boldsymbol{t}}^{(\Pi)}\left(\boldsymbol{u}^{(\Pi)}\right)$ is a nonlinear traction load on the Neumann boundary $\gamma_{\sigma}^{(I)}$ of body $\Omega^{(I)}$ with its unit outward surface normal $\boldsymbol{n}^{(\Pi)}$ given in the current configuration. The Neumann boundaries $\gamma_{\sigma}^{(\Pi)}$ are further specified in section 2.5. The vessel is embedded in spring boundary conditions (equations (1c) and (1d)) on the abluminal surface of the aorta $\Gamma_{\mathrm{ex}}^{\mathrm{Ao}}$ and the in- and outlet of the aorta $\Gamma_{\text {io }}^{\text {Ao }}$, respectively. $\hat{k}_{\text {ex }}$ and $\hat{k}_{\text {io }}$ are spring stiffnesses per unit reference area and $\boldsymbol{N}^{\mathrm{Ao}}$ is the unit outward surface normal of the reference configuration. Equation (1e) is a tied contact constraint on $\Gamma_{\mathrm{m}}=\Gamma_{1}^{\mathrm{G}} \cap \Gamma_{\mathrm{ex}}^{\mathrm{S}}$. The conditions (1f) are contact constraints in normal direction given in the form of Karush-Kuhn-Tucker conditions where $\boldsymbol{t}_{\mathrm{n}}$ is the normal contact traction at the contact interface and $g_{\mathrm{n}}$ is the gap function between the potential contact surfaces in the spatial configuration: the slave surface $\left(\gamma_{\mathrm{ex}}^{\mathrm{G}}\right)$ and the master surface $\left(\gamma_{1}^{\mathrm{Ao}}\right)$. The constraints $(1 \mathrm{~g})$ are frictional contact constraints according to Coulomb's law where $\mu \geq 0$ is the friction coefficient.

\subsection{D morphing algorithm based on control curves}

The presented in silico EVAR methodology heavily relies on a 3D morphing algorithm introduced in this section.

Morphing (Metamorphosis) is the process of continuously and smoothly transforming one object into another originally used in image processing (e.g. [6,51, $50]$ ). From a mechanical perspective the morphing process describes a gradual transition of a body $\tilde{\Omega}^{\mathrm{B}}(t) \subset \mathbb{R}^{3}$ from its initial configuration $\tilde{\Omega}_{\mathrm{I}}^{\mathrm{B}}=\tilde{\Omega}^{\mathrm{B}}\left(t_{\mathrm{I}}\right) \subset \mathbb{R}^{3}$ into the target configuration $\tilde{\Omega}_{\mathrm{T}}^{\mathrm{B}}=\tilde{\Omega}^{\mathrm{B}}\left(t_{\mathrm{T}}\right) \subset \mathbb{R}^{3}$. We restrict the morphing algorithm to discretized bodies described by $n_{\mathrm{B}}$ nodes with the coordinates $\tilde{\boldsymbol{x}}_{\mathrm{I}}^{i} \in \tilde{\Omega}_{\mathrm{I}}^{\mathrm{B}}$ and $\tilde{\boldsymbol{x}}_{\mathrm{T}}^{i} \in \tilde{\Omega}_{\mathrm{T}}^{\mathrm{B}}$, respectively, with $i=1,2, \ldots, n_{\mathrm{B}}$. The objective is to find all intermediate configurations $\tilde{\Omega}^{\mathrm{B}}(t) \subset \mathbb{R}^{3} \times \mathcal{T} \backslash\left\{t_{\mathrm{I}}, t_{\mathrm{T}}\right\}$ with $\mathcal{T}=\left[t_{\mathrm{I}}, t_{\mathrm{T}}\right]$ with the nodal coordinates $\tilde{\boldsymbol{x}}^{i}(t) \in \Omega^{\mathrm{B}}(t)$ satisfying the following conditions:

- Mechanical compatibility: Maintain the proper history of mechanical quantities with regard to the stress-free reference configuration.

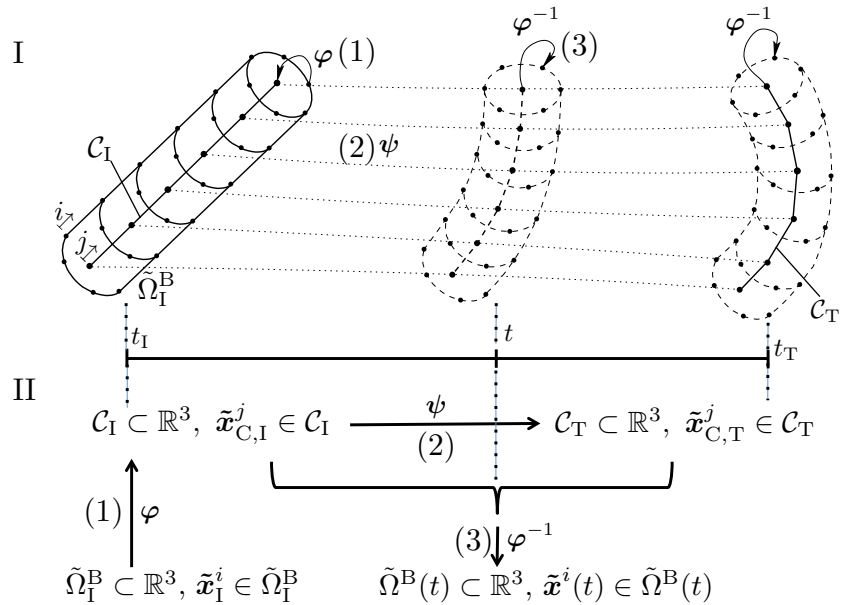

Fig. 2 Schematic overview (I) and algorithmic overiew (II) of the proposed morphing algorithm based on control curves consisting of three main steps: Initialization (1), path interpolation (2) and inverse mapping from $\mathcal{C}(t)$ to $\tilde{\Omega}^{\mathrm{B}}(t)(3)$; solid lines indicate the given configurations $\tilde{\Omega}_{\mathrm{I}}^{\mathrm{B}}, \mathcal{C}_{\mathrm{I}}$ and $\mathcal{C}_{\mathrm{T}}$; dashed lines refer to unknown configurations of $\tilde{\Omega}^{\mathrm{B}}$ and $\mathcal{C}$.

- Smoothness: The transition from $\tilde{\Omega}_{\mathrm{I}}^{\mathrm{B}}$ to $\tilde{\Omega}_{\mathrm{T}}^{\mathrm{B}}$ has to be smooth in space and in pseudo-time $t$.

We use the notation $(\tilde{\bullet})$ to indicate that the morphing algorithm is a pure geometrical problem. Nevertheless, for a consistent embedding into the mechanical framework of section 2.2, the first of the above conditions is required. To satisfy the second condition, morphing algorithms generally deal with two main issues:

- Point correspondence: Establishing a correspondence of each point (or node) of $\tilde{\Omega}_{\mathrm{I}}^{\mathrm{B}}$ to a point of $\tilde{\Omega}_{\mathrm{T}}^{\mathrm{B}}$.

- Path interpolation: Creating an interpolation along a pseudo-time $t$ between all corresponding points.

Especially the point correspondence problem can be challenging in general 3D problems. Hence, a common way is to reduce the dimensionality of the problem during the morphing process to 2D [44], 1D [6] or even 0D [11]. This approach called "field morphing" or "featurebased metamorphosis" was first introduced by Beier et al. [6] for 2D problems. The presented morphing algorithm strongly follows the idea of Beier et al. [6] and Lerios et al. [51] who reduced a multidimensional morphing object to $1 \mathrm{D}$ curves.

\subsubsection{Mapping between morphing object and control curve and definition of rotation minimizing local frames}

Given a piecewise linear approximation $\mathcal{C}(t) \subset \mathbb{R}^{3} \times \mathcal{T}$ to a $1 \mathrm{D}$ curve described by $n_{\mathrm{C}}$ discrete points in its 
initial configuration $\mathcal{C}_{\mathrm{I}}=\mathcal{C}\left(t_{\mathrm{I}}\right) \subset \mathbb{R}^{3}, \quad \tilde{\boldsymbol{x}}_{\mathrm{C}, \mathrm{I}}^{j} \in \mathcal{C}_{\mathrm{I}}$ and in its target configuration $\mathcal{C}_{\mathrm{T}}=\mathcal{C}\left(t_{\mathrm{T}}\right) \subset \mathbb{R}^{3}, \quad \tilde{\boldsymbol{x}}_{\mathrm{C}, \mathrm{T}}^{j} \in \mathcal{C}_{\mathrm{T}}$ with $j=1,2, \ldots, n_{\mathrm{C}}$, it is possible to define a unique deformation path of the $3 \mathrm{D}$ body from $\tilde{\Omega}_{\mathrm{I}}^{\mathrm{B}}$ to $\tilde{\Omega}_{\mathrm{T}}^{\mathrm{B}}$ based on the deformation of the curve from $\mathcal{C}_{\mathrm{I}}$ to $\mathcal{C}_{\mathrm{T}}$ (Figure $2 \mathrm{I})$. In doing so, we reduce the dimensionality of the problem from general $3 \mathrm{D}$ morphing objects $\tilde{\Omega}^{\mathrm{B}}$ to $1 \mathrm{D}$ curves $\mathcal{C}$. Hence, the two main issues of the morphing algorithm, point correspondence and path interpolation, only have to be established for 1D curves. In contrast, to classical morphing algorithms, the target configuration of the morphing object $\tilde{\Omega}_{\mathrm{T}}^{\mathrm{B}}$ is unknown. Only the initial configuration of the morphing object $\tilde{\Omega}_{\mathrm{I}}^{\mathrm{B}}$, the initial configuration of the curve $\mathcal{C}_{\mathrm{I}}$ and the target configuration of the curve $\mathcal{C}_{\mathrm{T}}$ are given.

Obviously, at any pseudo-time $t$ a map between the 1 D curve $\mathcal{C}$, in the following called control curve ${ }^{1}$, and the $3 \mathrm{D}$ body $\tilde{\Omega}^{\mathrm{B}}$ is required. Accordingly, the map

$\boldsymbol{\varphi}:\left\{\begin{array}{lll}\tilde{\Omega}^{\mathrm{B}}(t) & \rightarrow & \mathcal{C}(t) \\ \tilde{\boldsymbol{x}}^{i}(t) & \mapsto & \tilde{\boldsymbol{x}}_{\mathrm{C}}^{j}\left(\tilde{\boldsymbol{x}}^{i}(t)\right)\end{array}\right.$

and its inverse

$\varphi^{-1}:\left\{\begin{array}{lll}\mathcal{C}(t) & \rightarrow & \tilde{\Omega}^{\mathrm{B}}(t) \\ \tilde{\boldsymbol{x}}_{\mathrm{C}}^{j}(t) & \mapsto \quad \tilde{\boldsymbol{x}}^{i}\left(\tilde{\boldsymbol{x}}_{\mathrm{C}}^{j}(t)\right)\end{array}\right.$

are defined.

The mapping is based on the definition of $n_{\mathrm{C}}$ local, orthonormal coordinate frames $(\boldsymbol{t n} \boldsymbol{b})^{j}(t)$ at each point of the control curve tangentially aligned to the curve at any pseudo-time $t \in \mathcal{T}$, where $\boldsymbol{t}^{j}(t)$ is the unit tangent vector, $\boldsymbol{n}^{j}(t)$ the unit normal vector and $\boldsymbol{b}^{j}(t)$ the unit binormal vector (Figure 3 ).

The resulting description must be independent of the orientation of the control curve $\mathcal{C}(t)$ in $\mathbb{R}^{3}$. This means the local coordinate frames $(\boldsymbol{t n} \boldsymbol{b})^{j}(t)$ have to be uniquely defined for any configuration $\mathcal{C}(t)$ of the control curve at any pseudo-time $t \in \mathcal{T}$. The base vectors $\boldsymbol{t}^{j}(t), \boldsymbol{n}^{j}(t)$ and $\boldsymbol{b}^{j}(t)$ change over pseudo-time $t$, but for reasons of clarity we do not explicitly write the pseudo-time as function parameter in the following. Unless specified differently, the variables are valid for any pseudo-time $t \in \mathcal{T}$.

Whereas the definition of the unit tangent vector $\boldsymbol{t}^{j}=\frac{\mathcal{C}^{\prime} j}{\left\|\mathcal{C}^{\prime j}\right\|}$ is straight forward with $\mathcal{C}^{\prime j}$ being the first derivative of the curve at point $j$, the definition of $\boldsymbol{n}^{j}$ and $\boldsymbol{b}^{j}$ in general is not unique. In the present algorithm, we use a discrete form of a rotation minimizing frame (RMF) definition $[47,35,81]$. Such a set of $n_{\mathrm{C}}$

\footnotetext{
1 according to [6] who introduced the term "control primitives"
}

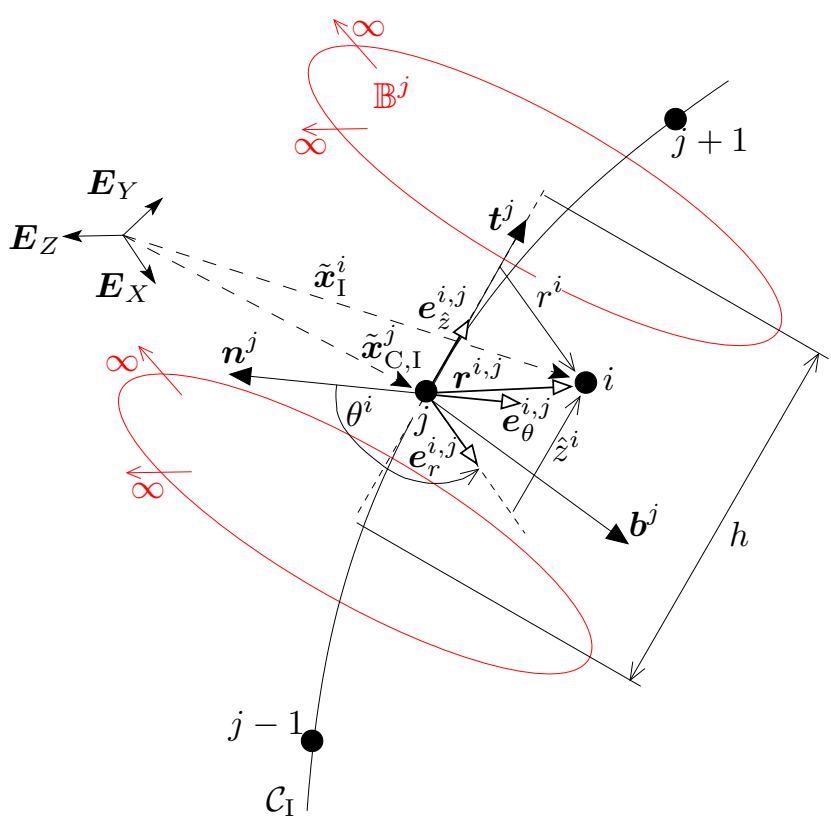

Fig. 3 Illustration of the local cylindrical coordinate system and the bounding box (red) around point $j$ of the control curve $\mathcal{C}_{\mathrm{I}}$

local frames $(\boldsymbol{t} \boldsymbol{n} \boldsymbol{b})^{j}$ is called rotation minimized if

$$
\begin{aligned}
& \boldsymbol{t}^{j}=\frac{\mathcal{C}^{\prime j}}{\left\|\mathcal{C}^{\prime j}\right\|}=\frac{\frac{1}{2}\left(\tilde{\boldsymbol{x}}_{\mathrm{C}}^{j+1}-\tilde{\boldsymbol{x}}_{\mathrm{C}}^{j-1}\right)}{\left\|\frac{1}{2}\left(\tilde{\boldsymbol{x}}_{\mathrm{C}}^{j+1}-\tilde{\boldsymbol{x}}_{\mathrm{C}}^{j-1}\right)\right\|}, \\
& \boldsymbol{n}^{j}=\underset{\boldsymbol{n}}{\operatorname{argmin}}\left(\sum_{j=1}^{n_{\mathrm{C}}}\left|\tau^{j}\right|\left\|\mathcal{C}^{\prime j}\right\|\right), \\
& \boldsymbol{n}^{j} \cdot \boldsymbol{t}^{j}=0, \\
& \boldsymbol{b}^{j}=\boldsymbol{t}^{j} \times \boldsymbol{n}^{j}, \quad \forall j=1,2, \ldots, n_{\mathrm{C}}
\end{aligned}
$$

holds. Equation (5b) states that for RMFs the total angle of rotation of the normal vectors $\boldsymbol{n}^{j}$ around curve $\mathcal{C}$ has to be minimal where the torsion

$\tau^{j}=-\boldsymbol{n}^{j} \cdot \boldsymbol{b}^{\prime}$

is a measure of the speed of rotation of the normal vector around the control curve at point $j[81,24]$ with $\boldsymbol{b}^{\prime j}$ being the first derivative of the binormal vector at point $j$. Equations (5c) and (5d) preserve the orthogonality property of the triads $(\boldsymbol{t} \boldsymbol{n} \boldsymbol{b})^{j}$. Accordingly, the triad $(\boldsymbol{t n} \boldsymbol{b})^{j}$ at any point $j$ is given by the solution of an initial-value problem [47], where $\boldsymbol{n}^{1}$ and $\boldsymbol{b}^{1}$ at point $j=1$ can be chosen arbitrarily ${ }^{2}$. A number of powerful methods to approximate the given problem are available $[47,8,81]$. We follow the approximation in Bloomenthal [8] which allows an explicit computation of all $n_{\mathrm{C}}$ local frames starting from a given frame spanned by

2 obviously the orthogonality property of the triad according to equations $(5 \mathrm{c})$ and $(5 \mathrm{~d})$ has to be preserved 
the unit vectors $\boldsymbol{t}^{1}, \boldsymbol{n}^{1}$ and $\boldsymbol{b}^{1}$ at point $j=1$. Accordingly, the triad at point $j$ is defined by

$\boldsymbol{t}^{j}=\frac{\mathcal{C}^{\prime j}}{\left\|\mathcal{C}^{\prime j}\right\|}=\frac{\frac{1}{2}\left(\tilde{\boldsymbol{x}}_{\mathrm{C}}^{j+1}-\tilde{\boldsymbol{x}}_{\mathrm{C}}^{j-1}\right)}{\left\|\frac{1}{2}\left(\tilde{\boldsymbol{x}}_{\mathrm{C}}^{j+1}-\tilde{\boldsymbol{x}}_{\mathrm{C}}^{j-1}\right)\right\|}$,

$\boldsymbol{n}^{j}=\frac{\boldsymbol{b}^{j-1} \times \boldsymbol{t}^{j}}{\left\|\boldsymbol{b}^{j-1} \times \boldsymbol{t}^{j}\right\|}$,

$\boldsymbol{b}^{j}=\frac{\boldsymbol{t}^{j} \times \boldsymbol{n}^{j}}{\left\|\boldsymbol{t}^{j} \times \boldsymbol{n}^{j}\right\|}, \quad \forall j=2,3, \ldots, n_{\mathrm{C}}$

In section 3.1, we demonstrate that this is an approximation of the exact initial-value problem of RMFs, which is largely sufficient for the presented application and also represents an exact solution of the RMFs for the trivial case of straight segments of curves $\left(\tau^{j}=0\right)$.

At each point $j$ of the control curve in the initial configuration with $\tilde{\boldsymbol{x}}_{\mathrm{C}, \mathrm{I}}^{j} \in \mathcal{C}_{\mathrm{I}}$, a semi-infinite bounding box $\mathbb{B}^{j} \subset \mathbb{R}^{3}$ spanned by two infinite planes in the local $(\boldsymbol{n b})^{j}\left(t_{\mathrm{I}}\right)$-plane with a search distance of $h$ is used to assign the nodes $i$ of the morphing object with $\tilde{\boldsymbol{x}}_{\mathrm{I}}^{i} \in \tilde{\Omega}_{\mathrm{I}}^{\mathrm{B}}$ to one point on the control curve $\mathcal{C}_{\mathrm{I}}$ (Figure 3 ). Accordingly, the semi-infinite bounding box $\mathbb{B}^{j}$ is infinite in radial direction but finite in tangential direction. All nodes $i$ of the morphing object $\tilde{\Omega}_{\mathrm{I}}^{\mathrm{B}}$ with $\tilde{x}_{\mathrm{I}}^{i} \in \mathbb{B}^{j}$ are assigned to point $j$ of the control curve $\mathcal{C}_{\mathrm{I}}$ and are put into the subset $\mathrm{A}_{\mathrm{I}}^{j} \subseteq \mathrm{A}_{\mathrm{I}}=\left\{1,2, \ldots, n_{\mathrm{B}}\right\}$ where

$$
\begin{gathered}
\bigcup_{j=1}^{n_{\mathrm{C}}} \mathrm{A}_{\mathrm{I}}^{j}=\mathrm{A}_{\mathrm{I}}, \\
\mathrm{A}_{\mathrm{I}}^{j} \cap \mathrm{A}_{\mathrm{I}}^{k}=\varnothing, \quad \forall k \neq j
\end{gathered}
$$

holds. Accordingly, all nodes $i=1,2, \ldots, n_{\mathrm{B}}$ of the morphing object $\tilde{\Omega}_{\mathrm{I}}^{\mathrm{B}}$ are assigned to a point $j=1,2, \ldots, n_{\mathrm{C}}$ of the control curve $\mathcal{C}_{\mathrm{I}}$ (equation (8a)). Further, equation $(8 \mathrm{~b})$ states that all subsets $\mathrm{A}_{\mathrm{I}}^{j}$ are pairwise disjoint sets, i.e. every node $i=1,2, \ldots, n_{\mathrm{B}}$ of the morphing object $\tilde{\Omega}_{\mathrm{I}}^{\mathrm{B}}$ is assigned to exactly one point $j=1,2, \ldots, n_{\mathrm{C}}$ of the control curve $\mathcal{C}_{\mathrm{I}}$. Within this section we restrict to a generic description of the morphing algorithm in which the subsets $\mathrm{A}^{j}$ are constant during the total morphing process, i.e.

$\mathrm{A}^{j}=\mathrm{A}_{\mathrm{I}}^{j}, \quad \forall j=1,2, \ldots, n_{\mathrm{C}}$.

However, in section 2.5, a different definition of $\mathrm{A}^{j}$ will be used for the in silico deployment of the SG.

The primary objective of the described morphing algorithm is to morph tube-like structures such as SGs which are rotationally symmetric about the longitudinal axis. Hence, we describe the position vectors $\tilde{\boldsymbol{x}}^{i}(t)$ of the morphing object in local cylindrical coordinate systems $\left(\boldsymbol{e}_{r} \boldsymbol{e}_{\theta} \boldsymbol{e}_{\hat{z}}\right)^{i, j}(t)$ (Figure 3 ). The relation between the previously introduced local frames $(\boldsymbol{t n} \boldsymbol{b})^{j}(t)$ and the local cylindrical coordinate systems $\left(\boldsymbol{e}_{r} \boldsymbol{e}_{\theta} \boldsymbol{e}_{\hat{z}}\right)^{i, j}(t)$ with the base vectors $\boldsymbol{e}_{r}^{i, j}(t), \boldsymbol{e}_{\theta}^{i, j}(t)$ and $\boldsymbol{e}_{\hat{z}}^{i, j}(t)$ is given by

$$
\begin{aligned}
& \boldsymbol{e}_{r}^{i, j}(t)=\boldsymbol{n}^{j}(t) \cos \left(\theta^{i}\right)+\boldsymbol{b}^{j}(t) \sin \left(\theta^{i}\right), \\
& \boldsymbol{e}_{\theta}^{i, j}(t)=-\boldsymbol{n}^{j}(t) \sin \left(\theta^{i}\right)+\boldsymbol{b}^{j}(t) \cos \left(\theta^{i}\right), \\
& \boldsymbol{e}_{\hat{z}}^{j}(t)=\boldsymbol{t}^{j}(t), \\
& \theta^{i} \in[0 ; 2 \pi], \quad \forall i \in \mathrm{A}^{j}, \quad j=1,2, \ldots, n_{\mathrm{C}} .
\end{aligned}
$$

Hence, the position vectors of the object $\tilde{\Omega}^{\mathrm{B}}$ to be morphed can be expressed by

$$
\begin{aligned}
\tilde{\boldsymbol{x}}^{i}(t) & =\tilde{\boldsymbol{x}}_{\mathrm{C}}^{j}(t)+\boldsymbol{r}^{i, j}(t) \\
& =\tilde{\boldsymbol{x}}_{\mathrm{C}}^{j}(t)+r^{i} \boldsymbol{e}_{r}^{i, j}(t)+\hat{z}^{i} \boldsymbol{e}_{\hat{z}}^{j}(t) \\
& \forall i \in \mathrm{A}^{j}, \quad j=1,2, \ldots, n_{\mathrm{C}} .
\end{aligned}
$$

Consequently, the position $\tilde{\boldsymbol{x}}^{i}$ of all nodes $i \in \mathrm{A}^{j}$ of the morphing object $\tilde{\Omega}^{\mathrm{B}}$ assigned to point $j$ of the control curve are described in the local cylindrical coordinate systems $\left(\boldsymbol{e}_{r} \boldsymbol{e}_{\theta} \boldsymbol{e}_{\hat{z}}\right)^{i, j}(t)$ according to equations (10a)-(10c) and (11) (Figure 3). Within this section we restrict to constant local cylindrical coordinates:

$r^{i}=r_{\mathrm{I}}^{i}, \quad \hat{z}^{i}=\hat{z}_{\mathrm{I}}^{i}, \theta^{i}=\theta_{\mathrm{I}}^{i}, \quad \forall i=1,2, \ldots, n_{\mathrm{B}}$.

This means during the total morphing process, the local cylindrical coordinates $r^{i}, \hat{z}^{i}$ and $\theta^{i}$ are equal to the local cylindrical coordinates $r_{\mathrm{I}}^{i}, \hat{z}_{\mathrm{I}}^{i}$ and $\theta_{\mathrm{I}}^{i}$ that correspond to the initial configuration of the morphing object $\tilde{\Omega}_{\mathrm{I}}^{\mathrm{B}}$. However, as the cylindrical base vectors $\boldsymbol{e}_{r}^{i, j}(t), \boldsymbol{e}_{\theta}^{i, j}(t)$ and $\boldsymbol{e}_{\hat{z}}^{i, j}(t)$ are aligned to the control curve $\mathcal{C}$ at any pseudo-time $t \in \mathcal{T}$, the base vectors $\boldsymbol{e}_{r}^{i, j}(t), \boldsymbol{e}_{\theta}^{i, j}(t)$ and $\boldsymbol{e}_{\hat{z}}^{i, j}(t)$ change during the morphing process. The change in the base vectors $\boldsymbol{e}_{r}^{i, j}(t), \boldsymbol{e}_{\theta}^{i, j}(t)$ and $\boldsymbol{e}_{\hat{z}_{\tilde{\Omega}}}^{i, j}(t)$ leads to a transformation of the morphing object $\tilde{\Omega}^{\mathrm{B}}(t)$ from $\tilde{\Omega}_{\mathrm{I}}^{\mathrm{B}}$ to $\tilde{\Omega}_{\mathrm{T}}^{\mathrm{B}}$ based on the transformation of the control curve $\mathcal{C}(t)$ from $\mathcal{C}_{\mathrm{I}}$ to $\mathcal{C}_{\mathrm{T}}$ (Figure 2 ). The transformation of the control curve $\mathcal{C}(t)$ over the pseudo-time $t$ will be further specified in section 2.3.2.

In section 2.5, we will use a different definition of equation (12) in order to change the radius of the SG during morphing operations in the in silico EVAR methodology.

\subsubsection{Point correspondence and path interpolation}

In the previous section, we reduced the dimensionality of the problem from general $3 \mathrm{D}$ morphing objects $\tilde{\Omega}^{\mathrm{B}}$ to a $1 \mathrm{D}$ control curve $\mathcal{C}$. Hence, point correspondence and path interpolation are only an issue of the $1 \mathrm{D}$ control curve. We use the point correspondence

$\tilde{\boldsymbol{x}}_{\mathrm{C}, \mathrm{I}}^{j} \leftrightarrow \tilde{\boldsymbol{x}}_{\mathrm{C}, \mathrm{T}}^{j}, \quad \forall j=1,2, \ldots, n_{\mathrm{C}}$ 
between the two known configurations $\mathcal{C}_{\mathrm{I}}$ and $\mathcal{C}_{\mathrm{T}}$ of the control curve, i.e. the first point of configuration $\mathcal{C}_{\mathrm{I}}$ corresponds to the first point of configuration $\mathcal{C}_{\mathrm{T}}$, the second point of configuration $\mathcal{C}_{\mathrm{I}}$ corresponds to the second point of configuration $\mathcal{C}_{\mathrm{T}}$ and so on.

Addressing the path interpolation problem, we look for a map $\boldsymbol{\psi}(t)$ that generates the intermediate configuration $\mathcal{C}(t)$ at any pseudo-time $t \in \mathcal{T} \backslash\left\{t_{\mathrm{I}}, t_{\mathrm{T}}\right\}$ of the two known configurations $\mathcal{C}_{\mathrm{I}}$ and $\mathcal{C}_{\mathrm{T}}$ :

$\boldsymbol{\psi}:\left\{\begin{array}{lll}\mathcal{C}_{\mathrm{I}}, \mathcal{C}_{\mathrm{T}} & \rightarrow & \mathcal{C}(t) \\ \tilde{\boldsymbol{x}}_{\mathrm{C}, \mathrm{I}}^{j}, \tilde{\boldsymbol{x}}_{\mathrm{C}, \mathrm{T}}^{j} & \mapsto & \tilde{\boldsymbol{x}}_{\mathrm{C}}^{j}\left(t, \tilde{\boldsymbol{x}}_{\mathrm{C}, \mathrm{I}}^{j}, \tilde{\boldsymbol{x}}_{\mathrm{C}, \mathrm{T}}^{j}\right) .\end{array}\right.$

We apply a simple linear path interpolation, defined by

$\tilde{\boldsymbol{x}}_{\mathrm{C}}^{j}(t):=\left(1-\frac{t-t_{\mathrm{I}}}{t_{\mathrm{T}}-t_{\mathrm{I}}}\right) \tilde{\boldsymbol{x}}_{\mathrm{C}, \mathrm{I}}^{j}+\frac{t-t_{\mathrm{I}}}{t_{\mathrm{T}}-t_{\mathrm{I}}} \tilde{\boldsymbol{x}}_{\mathrm{C}, \mathrm{T}}^{j}$,

$\forall j=1,2, \ldots, n_{\mathrm{C}}$,

despite some known shortcomings of linear interpolation between the initial configuration $\mathcal{C}_{\mathrm{I}}$ and the target configuration $\mathcal{C}_{\mathrm{T}}$. This approach might fail when the two known configurations $\mathcal{C}_{\mathrm{I}}$ and $\mathcal{C}_{\mathrm{T}}$ strongly differ in their orientation as this would lead to inappropriate intermediate configurations $\mathcal{C}(t)$ with possible selfintersections as described in detail in $[1,32]$.

To prevent the morphing object $\tilde{\Omega}^{\mathrm{B}}$ from spurious torsion between two different configurations $\tilde{\Omega}^{\mathrm{B}}(t)$ and $\tilde{\Omega}^{\mathrm{B}}(t+\Delta t)$, we apply the same explicit RMF scheme of equations (7a)-(7c) not only in space but also in pseudo-time. The RMF computation in pseudo-time $t$ is done only for the local coordinate system of the first point $(j=1)$ of the control curve $\mathcal{C}$ as all other RMFs for $j>1$ are explicitly computed by the RMF scheme in space (equations (7a)-(7c)). Hence, from arbitrarily chosen base vectors $\boldsymbol{n}^{1}\left(t_{\mathrm{I}}\right)$ and $\boldsymbol{b}^{1}\left(t_{\mathrm{I}}\right)$ at point $j=1$ of $\mathcal{C}_{\mathrm{I}}$, all other RMFs at point $j=1$ of $\mathcal{C}(t)$ with $t \in \mathcal{T} \backslash\left\{t_{\mathrm{I}}\right\}$ can be computed explicitly according to:

$$
\begin{aligned}
\boldsymbol{t}^{1}(t+\Delta t) & =\frac{\mathcal{C}(t+\Delta t)^{\prime 1}}{\left\|\mathcal{C}(t+\Delta t)^{\prime 1}\right\|}, \\
\boldsymbol{n}^{1}(t+\Delta t) & =\frac{\boldsymbol{b}^{1}(t) \times \boldsymbol{t}^{1}(t+\Delta t)}{\left\|\boldsymbol{b}^{1}(t) \times \boldsymbol{t}^{1}(t+\Delta t)\right\|}, \\
\boldsymbol{b}^{1}(t+\Delta t) & =\frac{\boldsymbol{t}^{1}(t+\Delta t) \times \boldsymbol{n}^{1}(t+\Delta t)}{\left\|\boldsymbol{t}^{1}(t+\Delta t) \times \boldsymbol{n}^{1}(t+\Delta t)\right\|},
\end{aligned}
$$

where $\mathcal{C}^{\prime 1}$ is the first spatial derivative of the control curve at point $j=1$.

Morphing algorithms might lead to self intersections leading to unphysical configurations of $\tilde{\Omega}^{\mathrm{B}}$. Especially, local self intersections have to be prevented. Local self intersections arise if the radius of curvature $r_{\kappa}^{j}$ of the control curve is smaller than the local radius $r^{i}$ of the morphing object at this point. Hence, during the whole morphing operation

$$
\begin{aligned}
f_{r}^{j}(t) & :=\max \left(\frac{r_{\kappa}^{j}(t)}{r^{i}}\right) \\
& =\max \left(\frac{1}{\left\|\boldsymbol{t}^{j+1}-\boldsymbol{t}^{j-1}\right\| r^{i}}\right)>\epsilon_{r}, \quad \forall i \in \mathrm{A}^{j}
\end{aligned}
$$

has to be guaranteed where $\epsilon_{\mathrm{r}}>1$ is a user-defined tolerance and $r_{\kappa}^{j}$ is the radius of curvature of the control curve at point $j$. In the limit case of a straight control curve, the radius of curvature $r_{\kappa}^{j}$ of the control curve would be infinity at any point $j=1,2, \ldots, n_{\mathrm{C}}$ and the condition of equation (17) would be satisfied for any radius $r^{i}$ of the morphing object.

As condition (17) would restrict the algorithm to relatively straight control curves, local smoothing of the curve is used which locally increases the radius of curvature $r_{\kappa}^{j}$ of the control curve such that condition (17) is satisfied. In the presented approach, local, iterative Laplacian smoothing according to

$$
\tilde{\boldsymbol{x}}_{\mathrm{C}}^{j}(t)=\left\{\begin{array}{lr}
\frac{1}{2}\left(\tilde{\boldsymbol{x}}_{\mathrm{C}}^{j-1}(t)+\tilde{\boldsymbol{x}}_{\mathrm{C}}^{j+1}(t)\right), \quad f_{r}^{j}(t) \leq \epsilon_{r} \\
\tilde{\boldsymbol{x}}_{\mathrm{C}}^{j}(t), \\
f_{r}^{j}(t)>\epsilon_{r}
\end{array},\right.
$$

is applied at locations where condition (17) is violated. The number of required smoothing iterations depends on the shape of the control curve. Laplacian smoothing induced shrinkage is removed by edge length scaling of all subsequent points of point $j$

$\tilde{\boldsymbol{x}}_{\mathrm{C}}^{k}(t)=\tilde{\boldsymbol{x}}_{\mathrm{C}}^{k-1}(t)+\Delta s^{j} \boldsymbol{t}^{j}, \quad \forall k \geq j$,

where $\Delta s^{j}$ is the mean edge length of the two adjacent edges of point $j$.

Due to the inherent map $\varphi^{-1}$ from equation (4) any smoothing of the control curve $\mathcal{C}$ results in a smoothed morphing object $\tilde{\Omega}^{\mathrm{B}}$ as well.

In summary, the proposed morphing algorithm computes all configurations $\tilde{\Omega}^{\mathrm{B}} \subset \mathbb{R}^{3} \times\left(t_{\mathrm{I}}, t_{\mathrm{T}}\right]$ of the morphing object from the given initial configuration of the morphing object $\tilde{\Omega}_{\mathrm{I}}^{\mathrm{B}}$, the initial configuration of the control curve $\mathcal{C}_{\mathrm{I}}$ and the target configuration of the control curve $\mathcal{C}_{\mathrm{T}}$ (Figure 2). The morphing algorithm is the solution to a purely geometric problem, i.e. the BVP of equations (1a)-(1g) does not have to be solved to obtain the deformation of the morphing object $\tilde{\Omega}^{\mathrm{B}}$. In section 2.5, the morphing algorithm will be applied in the in silico EVAR methodology where the SG will be the morphing object. 


\subsection{Constitutive modeling}

For all bodies $\Omega^{\mathrm{Ao}}, \Omega^{\mathrm{G}}$ and $\Omega^{\mathrm{S}}$ we assume the existence of an hyperelastic strain energy function (SEF) $\Psi$ such that $\boldsymbol{S}=2 \frac{\partial \Psi}{\partial \boldsymbol{C}}$ with the right Cauchy-Green tensor $\boldsymbol{C}=\boldsymbol{F}^{\mathrm{T}} \boldsymbol{F}$.

\subsubsection{Stent-graft}

In contrast to $[21,72]$, isotropy as in [46] is assumed for the graft made up of woven PET fabric which is sufficient in the first instance in which we are rather interested in the development of an in silico methodology. Hence, the graft material is modeled by the compressible neo-hookean model

$\Psi^{\mathrm{G}}\left(I_{1}, J\right)=\frac{c^{\mathrm{G}}}{\beta^{\mathrm{G}}}\left(J^{-2 \beta^{\mathrm{G}}}-1\right)+c^{\mathrm{G}}\left(I_{1}-3\right)$,

where $J=\operatorname{det}(\boldsymbol{F})$ and $I_{1}=\operatorname{tr}(\boldsymbol{C})$.

Stainless steel stents are modeled as elastic material $[21,72]$. The pure elastic regime can be assumed for selfexpandable stent-grafts as reported for instance in $[72$, 65]. We therefore apply the SEF

$\Psi^{\mathrm{S}}\left(I_{1}, J\right)=\frac{c^{\mathrm{S}}}{\beta^{\mathrm{S}}}\left(J^{-2 \beta^{\mathrm{S}}}-1\right)+c^{\mathrm{S}}\left(I_{1}-3\right)$

for this type of material behavior.

\subsubsection{Vessel}

Since arterial tissue is almost incompressible [10], an additive split of the corresponding SEF into a volumetric and an isochoric part is used. The isochoric-volumetric split enables the penalization of volumetric changes to sustain the almost incompressibility of the material. An Ogden volumetric SEF $[22,63]$ of type

$\Psi_{\mathrm{vol}}^{(\bullet)}(J)=\frac{K^{(\bullet)}}{4}\left(J^{2}-2 \ln J-1\right)$

is applied to all vessel constituents $(\bullet)$ considered in the model, i.e. for the SEF of "healthy" aortic wall (AA), "aneurysmatic" aortic wall (AAA), ILT and calcifications (calc). $K$ is the volumetric bulk modulus which is chosen sufficiently large to sustain almost incompressibility.

Different constitutive models are used for the "healthy" and the "aneurysmatic" parts of the vessel wall as substantial differences in the material behavior of "healthy" aortic walls and "aneurysmatic" aortic walls can be identified [61]. The "healthy" abdominal aortic wall is described by the anisotropic, hyperelastic two-fiber model proposed by Gasser et al. [29]. The isochoric SEF is a superposition of an isotropic ground substance $\Psi_{\text {iso }}^{\mathrm{AA}}$ and an anisotropic part $\Psi_{\mathrm{fi}}^{\mathrm{AA}}$ considering the collagen fibers of the vessel. The total SEF of the "healthy" vessel wall reads

$$
\begin{aligned}
& \Psi^{\mathrm{AA}}\left(\bar{I}_{1}, \bar{I}_{4}, \bar{I}_{6}, J\right) \\
& =\Psi_{\mathrm{iso}}^{\mathrm{AA}}\left(\bar{I}_{1}\right)+\Psi_{\mathrm{fi}}^{\mathrm{AA}}\left(\bar{I}_{4}, \bar{I}_{6}\right)+\Psi_{\mathrm{vol}}^{\mathrm{AA}}(J) \\
& =c^{\mathrm{AA}}\left(\bar{I}_{1}-3\right)+\frac{k_{1}}{2 k_{2}} \sum_{i=4,6}\left(e^{k_{2}\left[\kappa \bar{I}_{1}+(1-3 \kappa) \bar{I}_{i}-1\right]^{2}}-1\right) \\
& \\
& \quad+\Psi_{\mathrm{vol}}^{\mathrm{AA}}(J)
\end{aligned}
$$

where $\bar{I}_{1}=J^{-2 / 3} \operatorname{tr}(\boldsymbol{C})$ refers to the first modified invariant of the right Cauchy-Green strain tensor $\boldsymbol{C}$ and $\bar{I}_{4}$ as well as $\bar{I}_{6}$ to the squares of the stretches in fiber direction as defined in [29]. $\kappa$ is the transversely isotropic dispersion parameter, $c^{\mathrm{AA}}$ is the isotropic shear modulus and $k_{1}$ as well as $k_{2}$ are fiber-specific stiffness parameters.

In the "aneurysmatic" abdominal aortic wall, the collagen fibers are more dispersed and isotropy is a widely used model assumption $[70,71,54,31]$. Here, the following SEF is applied for the "aneurysmatic" aortic wall

$$
\begin{aligned}
& \Psi^{\mathrm{AAA}}\left(\bar{I}_{1}, J\right) \\
& =\Psi_{\mathrm{iso}}^{\mathrm{AAA}}\left(\bar{I}_{1}\right)+\Psi_{\mathrm{vol}}^{\mathrm{AAA}}(J) \\
& =a\left(\bar{I}_{1}-3\right)+b\left(\bar{I}_{1}-3\right)^{2}+\Psi_{\mathrm{vol}}^{\mathrm{AAA}}(J),
\end{aligned}
$$

where $a$ and $b$ are material parameters according to Raghavan and Vorp [70].

A geometrical parameter

$\lambda(d)= \begin{cases}0, & d<d_{0}, \\ \frac{1}{2}\left(1-\cos \left(\pi \frac{d-d_{0}}{d_{1}-d_{0}}\right)\right), & d_{0} \leq d \leq d_{1}, \\ 1, & d>d_{1}\end{cases}$

is defined to blend smoothly between "healthy" and "aneurysmatic" aortic wall:

$\Psi^{\text {wall }}=(1-\lambda(d)) \Psi^{\mathrm{AA}}+\lambda(d) \Psi^{\mathrm{AAA}}$.

$\lambda(d) \in[0 ; 1]$ is a function of the local diameter $d$ of the vessel. The bounds $d_{0}$ and $d_{1}$ are chosen as $d_{0}=1.2 d^{\mathrm{AA}}$ and $d_{1}=1.5 d^{\mathrm{AA}}$ with $d^{\mathrm{AA}}$ being the mean diameter of the vessel in the most proximal region which is assumed to be in "healthy" state. Accordingly, regions of the vessel with a local diameter $d$ smaller than $d_{0}$ are fully described by the SEF $\Psi_{A A}$ of a "healthy" vessel whereas regions with a local diameter $d$ larger than $d_{1}$ are fully described by the SEF $\Psi^{\mathrm{AAA}}$ of an "aneurysmatic" vessel. In-between a transition zone of partly "aneurysmatic" vessel material arises as shown in Figure $7 \mathrm{I}$.

ILT is a fibrin structure incorporated with blood cells and proteins, platelets and cellular debris [80] with 
macroscopically isotropic mechanical behavior $[28,80$, 78]. The SEF

$$
\begin{aligned}
\Psi^{\mathrm{ILT}}\left(\bar{I}_{1}, \bar{I}_{2}, J\right) & =\Psi_{\mathrm{iso}}^{\mathrm{ILT}}\left(\bar{I}_{1}, \bar{I}_{2}\right)+\Psi_{\mathrm{vol}}^{\mathrm{ILT}}(J) \\
& =c^{\mathrm{ILT}}\left(\bar{I}_{1}^{2}-2 \bar{I}_{2}-3\right)+\Psi_{\mathrm{vol}}^{\mathrm{ILT}}(J)
\end{aligned}
$$

proposed by Gasser et al. [28] is utilized to model the mechanical properties of ILT. $\bar{I}_{2}=J^{-4 / 3} \operatorname{tr}(\boldsymbol{C}) \operatorname{det}(\boldsymbol{C})$ refers to the second modified invariant of the right Cauchy-Green strain tensor $\boldsymbol{C}$. The constitutive model resolves the material properties of three different ILT layers with decreasing stiffness $c^{\mathrm{ILT}}$ : luminal $c_{\text {lum }}$, medial $c_{\text {med }}$ and abluminal $c_{\text {abl }}$. The material parameter $c^{\mathrm{ILT}}$ is piecewise linearly interpolated between these distinct ILT layers from the luminal to the abluminal surface of the ILT.

Since calcifications can have a very irregular shape and since they can be located at a large number of separated spots, modeling and discretization of calcifications as separate constituents is not practicable. Instead, calcifications are considered implicitly within the domains of ILT and aortic wall. The SEF representing the mechanical behavior of calcifications is superimposed to the SEF of ILT and the aortic wall, respectively.

$$
\begin{aligned}
\hat{\Psi}^{\mathrm{ILT}} & =\Psi^{\mathrm{ILT}}+\Psi^{\mathrm{calc}}, \\
\hat{\Psi}^{\mathrm{wall}} & =\Psi^{\mathrm{wall}}+\Psi^{\mathrm{calc}} .
\end{aligned}
$$

The SEF $\hat{\Psi}^{\mathrm{ILT}}$ and $\hat{\Psi}^{\text {wall }}$ consider the material behavior of calcifications implicitly within the aortic tissue. The SEF $\Psi^{\text {calc }}$ is a function of the local Hounsfield units (HU) of the vessel which generally are extracted from CT images. The almost linear material behavior of calcifications reported in [53] is modeled by a neo-Hookean material for the isochoric component of the SEFgray

$$
\begin{aligned}
\Psi^{\text {calc }}\left(\bar{I}_{1}, J\right) & =\Psi_{\mathrm{iso}}^{\text {calc }}\left(\bar{I}_{1}\right)+\Psi_{\mathrm{vol}}^{\text {calc }}(J) \\
& =c^{\text {calc }}\left(\bar{I}_{1}-3\right)+\Psi_{\mathrm{vol}}^{\text {calc }}(J),
\end{aligned}
$$

where $c^{\text {calc }}$ is the additional stiffness of the calcified regions that is added to the base material of ILT and vessel wall, respectively. As stated for instance in [53], "pure calcifications" and "calcified tissue" have to be distinguished, the latter one meaning a compound of aortic tissue and dispersed calcifications. This motivates the definition of $\alpha$ as a smooth transition from "noncalcified" to "pure calcification" with

$$
\begin{aligned}
c^{\text {calc }}(h) & = \begin{cases}0, & h<h_{0}, \\
f^{\text {calc }}(h), & h_{0} \leq h \leq h_{1}, \\
\hat{c}^{\text {calc }}, & h>h_{0}\end{cases} \\
f^{\text {calc }}(h) & =\frac{1}{2} \hat{c}^{\text {calc }}\left(1-\cos \left(\pi \frac{h-h_{0}}{h_{1}-h_{0}}\right)\right),
\end{aligned}
$$

Table 2 Material parameters of vessel and SG constitutive models.

\begin{tabular}{clcl}
\hline \hline Stent & {$[21]$} & Graft & {$[72]$} \\
$c^{\mathrm{S}}[\mathrm{GPa}]$ & 40.4 & $c^{\mathrm{G}}[\mathrm{GPa}]$ & $0.050^{\mathrm{a}}$ \\
$\beta^{\mathrm{S}}[-]$ & 0.75 & $\beta^{\mathrm{G}}[-]$ & 2.625 \\
Healthy aortic wall & {$[37]$} & AAA wall & {$[70]$} \\
$c^{\mathrm{AA}}[\mathrm{kPa}]$ & 100.9 & $a[\mathrm{kPa}]$ & 174.0 \\
$k_{1}[\mathrm{kPa}]$ & 4070.0 & $b[\mathrm{kPa}]$ & 1881.0 \\
$k_{2}[-]$ & 166.5 & & \\
$\kappa[-]$ & 0.16 & & \\
$\mathbf{I L T}$ & {$[28]$} & Calcification & {$[53]$} \\
$c_{\text {lum }}[\mathrm{kPa}]$ & 2.62 & $\hat{c}^{\text {calc }}[\mathrm{kPa}]$ & 8929.0 \\
$c_{\text {med }}[\mathrm{kPa}]$ & 1.98 & & \\
$c_{\text {abl }}[\mathrm{kPa}]$ & 1.73 & & \\
Surrounding tissue & {$[57]$} & & \\
$\hat{k}_{\text {ex }}[\mathrm{kPa} / \mathrm{mm}]$ & 2.0 & & \\
$\hat{k}_{\text {io }}[\mathrm{kPa} / \mathrm{mm}]$ & 4.0 & & \\
\hline \hline
\end{tabular}

a mean of Young's modulus in axial and in circumferential direction from Roy et al. [72]

where $h$ is the local HU value, $h_{0}$ is the $\mathrm{HU}$ value at which the transition from noncalcified aortic tissue to calcified tissue starts, $h_{1}$ is the HU value above which the material is considered as pure calcification.

Table 2 summarizes the material parameters of all proposed constitutive laws.

\subsection{In silico EVAR methodology}

Given the vessel as well as the SG in the undeformed configuration, we aim to find the final deployed SG configuration within the elastically deformable vessel under physiologically meaningful conditions, where nonetheless a pseudo-stationary problem is assumed. Investigations on the influence of the dynamics of the pulsatory blood flow are not part of the present study. The presented methodology of in silico EVAR is subdivided into the steps: Vessel prestressing, Stent predeformation, SG placement and $S G$ deployment. Subsequent $S G$ parameter continuation is used to continuously change the SG diameter starting from an already deployed SG configuration.

In the in silico EVAR methodology we make use of a change in the reference configuration, i.e. the initial configuration not necessarily has to be equivalent to the stress-free reference configuration. Depending on the particular stage of the in silico EVAR methodology, the stress-free state is defined by a different reference configuration. We define six distinct configurations of the SG where the configurations visualized in Figure 4I-III describe stress-free reference configurations and the configurations visualized in Figure 4IV-VI describe current configurations. All reference configurations are denoted by the subscript $(\bullet)_{0}$ whereas all other configurations are current configurations. Further, 


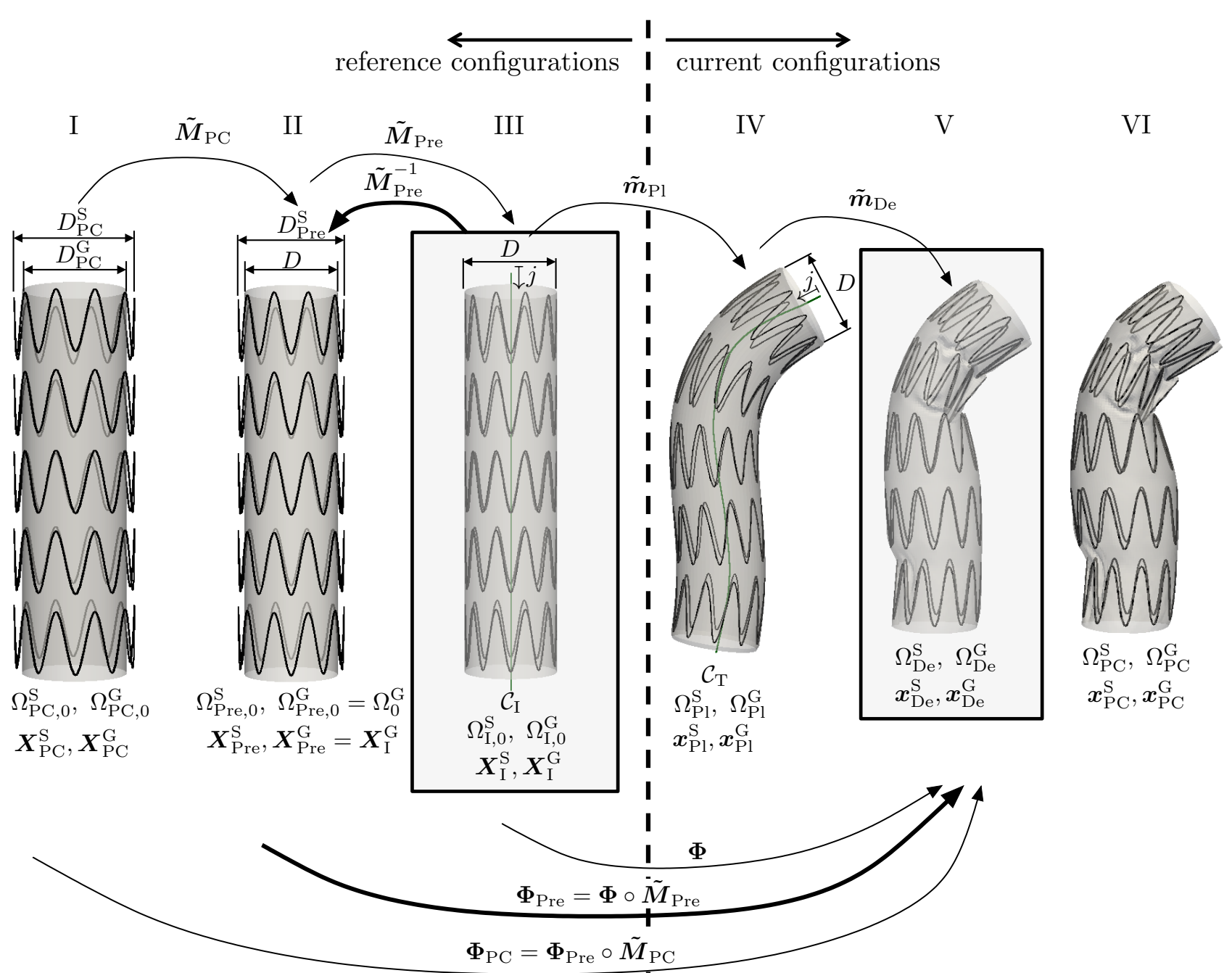

Fig. 4 Overview of configurations of the SG $\Omega_{(\Upsilon)}^{\{\mathrm{S}, \mathrm{G}\}}$ with $\Upsilon=\{\mathrm{I}, \mathrm{Pre}, \mathrm{Pl}, \mathrm{De}, \mathrm{PC}\}$ during the in silico EVAR methodology (initial state (I), stent predeformation (Pre), SG placement (Pl) and SG deployment (De)) and SG parameter continuation $(\mathrm{PC})$; subscript $(\bullet)_{0}$ indicates a stress-free reference configuration described by the reference coordinates $\boldsymbol{X}$; configurations without subscript $(\bullet)_{0}$ are current configurations described by the current coordinates $\boldsymbol{x}$; diffeomorphic morphing maps $\tilde{\boldsymbol{M}}$ and $\tilde{\boldsymbol{m}}$ as well as deformation maps $\boldsymbol{\Phi}, \boldsymbol{\Phi}_{\mathrm{Pre}}$ and $\boldsymbol{\Phi}_{\mathrm{PC}}$ define the relation between the different configurations of the SG; initial configuration $\mathcal{C}_{\mathrm{I}}$ and target configuration $\mathcal{C}_{\mathrm{T}}$ of the control curve (green).

the subscript $(\bullet)_{\text {Pre }}$ denotes the stent predeformation, $(\bullet)_{\mathrm{Pl}}$ denotes the SG placement and $(\bullet)_{\text {De }}$ the SG deployment. The subscript $(\bullet)_{\mathrm{PC}}$ describes the parameter continuation approach which is used to change the diameter of the SG, i.e. the degree of SG oversizing. Accordingly, $\Omega_{\mathrm{Pre}, 0}^{\{\mathrm{S}, \mathrm{G}\}}$ describes the stress-free reference configurations of stent (superscript $(\bullet)^{\mathrm{S}}$ ) and graft (superscript $(\bullet)^{\mathrm{G}}$ ) after the stent predeformation has been applied to the SG. $\Omega_{\mathrm{Pl}}^{\{\mathrm{S}, \mathrm{G}\}}$ are the current configurations of stent and graft after the in silico SG placement has been performed and $\Omega_{\mathrm{De}}^{\{\mathrm{S}, \mathrm{G}\}}$ are the current configurations of stent and graft after the in silico $\mathrm{SG}$ deployment has been done. $\Omega_{\mathrm{PC}, 0}^{\{\mathrm{S}, \mathrm{G}\}}$ are the stress-free reference configurations of stent and graft and $\Omega_{\mathrm{PC}}^{\{\mathrm{S}, \mathrm{G}\}}$ are the current configurations of stent and graft after the SG diameter has been changed by the parameter continuation approach (Figure 4).

The initial reference configurations of stent and graft $\Omega_{\mathrm{I}, 0}^{\{\mathrm{S}, \mathrm{G}\}}$, i.e. the discretized stent and graft configurations with which the in silico EVAR methodology starts, are visualized in Figure 4III. The final deployed configurations of stent and graft $\Omega_{\mathrm{De}}^{\{\mathrm{S}, \mathrm{G}\}}$, i.e. the result of the in silico EVAR methodology without parameter continuation, are visualized in Figure $4 \mathrm{~V}$.

We describe the position vector of a reference configuration by the capital letter $\boldsymbol{X}$ and the position vector of a current configuration by the small letter $\boldsymbol{x}$. At any stage of the in silico EVAR methodology, the current configurations $\Omega_{(\Upsilon)}^{(\Pi)}$ with $\Pi=\{$ Ao, G, S $\}$ and $\Upsilon=\{\mathrm{I}, \mathrm{Pre}, \mathrm{Pl}, \mathrm{De}, \mathrm{PC}\}$ are related to the correspond- 
ing stress-free reference configurations $\Omega_{(\Upsilon), 0}^{(\Pi)}$ via the mapping $\boldsymbol{\Phi}$ (equation (2)) based on the BVP of equations (1a)-(1g). However, as in the in silico EVAR methodology the stress-free reference configurations of stent and graft are modified, we additionally define the mappings $\boldsymbol{\Phi}_{\text {Pre }}$ and $\boldsymbol{\Phi}_{\text {PC }}$. $\boldsymbol{\Phi}_{\text {Pre }}$ relates the current configurations to the corresponding stress-free reference configurations after the stent predeformation has been applied. $\boldsymbol{\Phi}_{\mathrm{PC}}$ relates the current configurations to the corresponding stress-free reference configurations after the parameter continuation has been applied (Figure 4).

The in silico EVAR methodology is strongly based on the $3 \mathrm{D}$ morphing algorithm presented in section 2.3. In contrast to section 2.3, now the morphing operations are applied to stent and graft, i.e. the morphing operations are applied to physical bodies underlying the BVP of equations (1a)-(1g). The methodology is governed by a series of prescribed morphing maps $\tilde{M}$ and $\tilde{\boldsymbol{m}}$. In section 2.3 we have introduced the pseudotime $t \in\left[t_{\mathrm{I}}, t_{\mathrm{T}}\right]$ which describes the interpolation between the two known configurations of the control curve $\mathcal{C}_{\mathrm{I}}$ and $\mathcal{C}_{\mathrm{T}}$ (equation (15)). This means the pseudotime $t$ describes the deformation of the morphing object according to the evolution of the control curve $\mathcal{C}(t)$. From now on, $\mathcal{C}_{\mathrm{I}}$ is the centerline of the initial configuration of the SG $\Omega_{\mathrm{I}, 0}^{\{\mathrm{S}, \mathrm{G}\}}$ and $\mathcal{C}_{\mathrm{T}}$ is the centerline of the initial, undeformed configuration of the vessel $\Omega_{I, 0}^{\text {Ao }}$. Both, $\mathcal{C}_{\mathrm{I}}$ and $\mathcal{C}_{\mathrm{T}}$ are described by $n_{\mathrm{C}}$ points. Independently of the control curve induced deformation of the morphing object according to the pseudo-time $t$, we define a prescribed change in the radius of the morphing object and a prescribed release of the morphing constraints.

A prescribed change in the radius of the morphing object by the factor $\Delta r$ is induced by redefining equation (12) such that the local radius $r^{i}$ of the morphing object is given by

$r^{i}=r_{\mathrm{I}}^{i}+\Delta r, \quad \forall i=1,2, \ldots, n_{\mathrm{B}}$,

where $r_{\mathrm{I}}^{i}$ are the local radii of the morphing object in the initial state corresponding to the initial configuration $\mathcal{C}_{\mathrm{I}}$ of the control curve and $n_{\mathrm{B}}$ is the number of nodes of the morphing object. This prescribed change in the radius is used in the $S G$ placement substep to apply radial crimping to the $\mathrm{SG}$ such that after morphing the SG inside the vessel, it will not touch the luminal vessel surface before contact is turned on. Further, this prescribed change in the radius of the morphing object will be used in the substeps: stent predeformation and $S G$ parameter continuation.

A prescribed release of the morphing constraints is induced by the redefinition of the morphing sets $A^{j}$ with $j=1,2, \ldots, n_{\mathrm{C}}$ (equation (9)). If the deformation of a node of the morphing object is prescribed by the morphing induced deformation or not, depends on whether this node is part of the total set of morphed nodes

$\mathrm{A}=\bigcup_{j=1}^{n_{\mathrm{C}}} \mathrm{A}^{j}$,

i.e. whether this nodes is assigned to any of the points of the control curve $\mathcal{C}$ (cf. equation (8a)). This means that by emptying the sets $\mathrm{A}^{j}$ a prescribed release of the morphing constraints is possible. A prescribed release of the morphing constraints will be used in the following substep: $S G$ deployment.

As the three morphing operations, control curve induced deformation, radius change in the morphing object and release of the morphing constraints shall be independent of each other, we define a second pseudotime $\xi \in[0 ; 1]$ which defines the progress of the applied morphing maps $\tilde{\boldsymbol{M}}(\xi)$ and $\tilde{\boldsymbol{m}}(\xi)$. Hence, $t(\xi)$ defining the control curve induced deformation, $\Delta r(\xi)$ defining the radius change in the morphing object and $\mathrm{A}^{j}(\xi)$ defining the release of the morphing constraints are functions of the pseudo-time $\xi$, i.e. they depend on the progress of the applied morphing map.

The change in the reference configuration from $\left.\Omega_{(\Upsilon), 0}^{\{\mathrm{S}, \mathrm{G}\}}\right|_{\xi=0}$ to $\left.\Omega_{(\Upsilon), 0}^{\{\mathrm{S}, \mathrm{G}\}}\right|_{\xi=1}$ with $\Upsilon=\{\mathrm{I}$, Pre, Pl, De, PC $\}$ by a geometrically prescribed morphing process is defined by the diffeomorphic morphing map

$$
\begin{aligned}
\tilde{\boldsymbol{M}}\left(t(\xi), \Delta r(\xi), \mathrm{A}^{j}(\xi)\right): & \left\{\begin{array}{lll}
\left.\Omega_{(\Upsilon), 0}^{\{\mathrm{S}, \mathrm{G}\}}\right|_{\xi=0} & \rightarrow & \left.\Omega_{(\Upsilon), 0}^{\{\mathrm{S}, \mathrm{G}\}}\right|_{\xi=1} \\
\left.\boldsymbol{X}_{(\Upsilon)}^{\{\mathrm{S}, \mathrm{G}\}}\right|_{\xi=0} & \mapsto & \left.\boldsymbol{X}_{(\Upsilon)}^{\{\mathrm{S}, \mathrm{G}\}}\right|_{\xi=1},
\end{array}\right.
\end{aligned}
$$

where $\left.\quad \Omega_{(\Upsilon), 0}^{\{\mathrm{S}, \mathrm{G}\}}\right|_{\xi=0},\left.\left.\quad \boldsymbol{X}_{(\Upsilon)}^{\{\mathrm{S}, \mathrm{G}\}}\right|_{\xi=0} \in \Omega_{(\Upsilon), 0}^{\{\mathrm{S}, \mathrm{G}\}}\right|_{\xi=0}$ describes the stress-free reference configuration at the beginning of the morphing process $\left(\left.(\bullet)\right|_{\xi=0}\right)$, i.e. at $\xi=0$. At the end of the morphing process $\left(\left.(\bullet)\right|_{\xi=1}\right)$, the reference configuration is given by $\left.\Omega_{(\Upsilon), 0}^{\{\mathrm{S}, \mathrm{G}\}}\right|_{\xi=1},\left.\left.\boldsymbol{X}_{(\Upsilon)}^{\{\mathrm{S}, \mathrm{G}\}}\right|_{\xi=1} \in \Omega_{(\Upsilon), 0}^{\{\mathrm{S}, \mathrm{G}\}}\right|_{\xi=1}$. Inbetween, i.e. for $\xi \in(0,1)$, the reference configuration is determined by an interpolation between the configurations $\left.\Omega_{(\Upsilon), 0}^{\{\mathrm{S}, \mathrm{G}\}}\right|_{\xi=0}$ and $\left.\Omega_{(\Upsilon), 0}^{\{\mathrm{S}, \mathrm{G}\}}\right|_{\xi=1}$ which is driven by $t(\xi)$, $\Delta r(\xi)$ and $\mathrm{A}^{j}(\xi)$.

Analogously, the change in the current configuration from $\left.\Omega_{(\Upsilon)}^{\{\mathrm{S}, \mathrm{G}\}}\right|_{\xi=0}$ to $\left.\Omega_{(\Upsilon)}^{\{\mathrm{S}, \mathrm{G}\}}\right|_{\xi=1}$ by a geometrically prescribed morphing process is defined by the diffeomorphic morphing map

$$
\begin{aligned}
\tilde{\boldsymbol{m}}\left(t(\xi), \Delta r(\xi), \mathrm{A}^{j}(\xi)\right): & \left\{\begin{array}{lll}
\left.\Omega_{(\Upsilon)}^{\{\mathrm{S}, \mathrm{G}\}}\right|_{\xi=0} & \rightarrow & \left.\Omega_{(\Upsilon)}^{\{\mathrm{S}, \mathrm{G}\}}\right|_{\xi=1} \\
\left.\boldsymbol{x}_{(\Upsilon)}^{\{\mathrm{S}, \mathrm{G}\}}\right|_{\xi=0} & \mapsto & \left.\boldsymbol{x}_{(\Upsilon)}^{\{\mathrm{S}, \mathrm{G}\}}\right|_{\xi=1},
\end{array}\right.
\end{aligned}
$$


where $\left.\Omega_{(\Upsilon)}^{\{\mathrm{S}, \mathrm{G}\}}\right|_{\xi=0},\left.\left.\boldsymbol{x}_{(\Upsilon)}^{\{\mathrm{S}, \mathrm{G}\}}\right|_{\xi=0} \in \Omega_{(\Upsilon)}^{\{\mathrm{S}, \mathrm{G}\}}\right|_{\xi=0}$ describes the current configuration at the beginning of the morphing process $\left(\left.(\bullet)\right|_{\xi=0}\right)$. At the end of the morphing process $\left(\left.(\bullet)\right|_{\xi=1}\right)$, the current configuration is determined by $\left.\Omega_{(\Upsilon)}^{\{\mathrm{S}, \mathrm{G}\}}\right|_{\xi=1} ^{\xi=1},\left.\left.\boldsymbol{x}_{(\Upsilon)}^{\{\mathrm{S}, \mathrm{G}\}}\right|_{\xi=1} \in \Omega_{(\Upsilon)}^{\{\mathrm{S}, \mathrm{G}\}}\right|_{\xi=1}$. In-between, i.e. for $\xi \in(0,1)$, the current configuration is determined by a $t(\xi)-\Delta r(\xi)-\mathrm{A}^{j}(\xi)$-driven interpolation between the configurations $\left.\Omega_{(\Upsilon)}^{\{\mathrm{S}, \mathrm{G}\}}\right|_{\xi=0}$ and $\left.\Omega_{(\Upsilon)}^{\{\mathrm{S}, \mathrm{G}\}}\right|_{\xi=1}$. These abstract definitions of $\tilde{\boldsymbol{M}}(\xi)$ changing a reference configuration and $\tilde{\boldsymbol{m}}(\xi)$ changing a current configuration will be further specified in the following subsections 2.5.2 to 2.5.5. To clearly distinguish between a change in the current configuration and a change in the reference configuration, we denote any morphing map that changes the current configuration by a small letter $\tilde{\boldsymbol{m}}$ and any morphing map that changes the reference configuration by a capital letter $\tilde{\boldsymbol{M}}$.

The structure of the following subsections is divided into the single substeps of the in silico EVAR methodology: Vessel prestressing, Stent predeformation, SG placement and $S G$ deployment which are visualized in Figure 5. In section 2.5.5, we introduce a parameter continuation approach to continuously change the SG diameter starting from the configuration of a deployed SG. This continuous change in the SG diameter is a valuable tool when addressing the issue of the optimal degree of SG oversizing.

\subsubsection{Vessel prestressing}

In patient-specific modeling the geometry naturally is reconstructed from in vivo medical imaging such as CT scans and therefore involves an initial geometric configuration that is not stress-free. In order to initialize the model to this stressed configuration, we apply a Modified Update Lagrangian Formulation proposed in [30] and prestress the vessel to the diastolic pressure $p_{\mathrm{d}}=80 \mathrm{mmHg}$ (Figure 5I).

\subsubsection{Stent predeformation}

Stents of most marketed SGs are manufactured with a diameter $D_{\text {Pre }}^{\mathrm{S}}>D$ where $D$ is the nominal diameter of the SG (Figure 5II or cf. [72]). The degree of stent predeformation $\frac{D_{\text {Pre }}^{\mathrm{S}}}{D}$ depends on the SG type and manufacturer. In our approach, initially the SG geometry is generated with $D^{\mathrm{S}}=D^{\mathrm{G}}=D$ (configuration $\Omega_{\mathrm{I}, 0}^{\{\mathrm{S}, \mathrm{G}\}}$ ), i.e. stent diameter $D^{\mathrm{S}}$ as well as graft $D^{\mathrm{G}}$ are equivalent to the nominal diameter $D$ of the SG. The stent is attached to the graft by applying mortar based mesh tying on the common interface $\Gamma_{\mathrm{m}}=\Gamma_{\mathrm{l}}^{\mathrm{G}} \cap \Gamma_{\mathrm{ex}}^{\mathrm{S}}$ of stent and
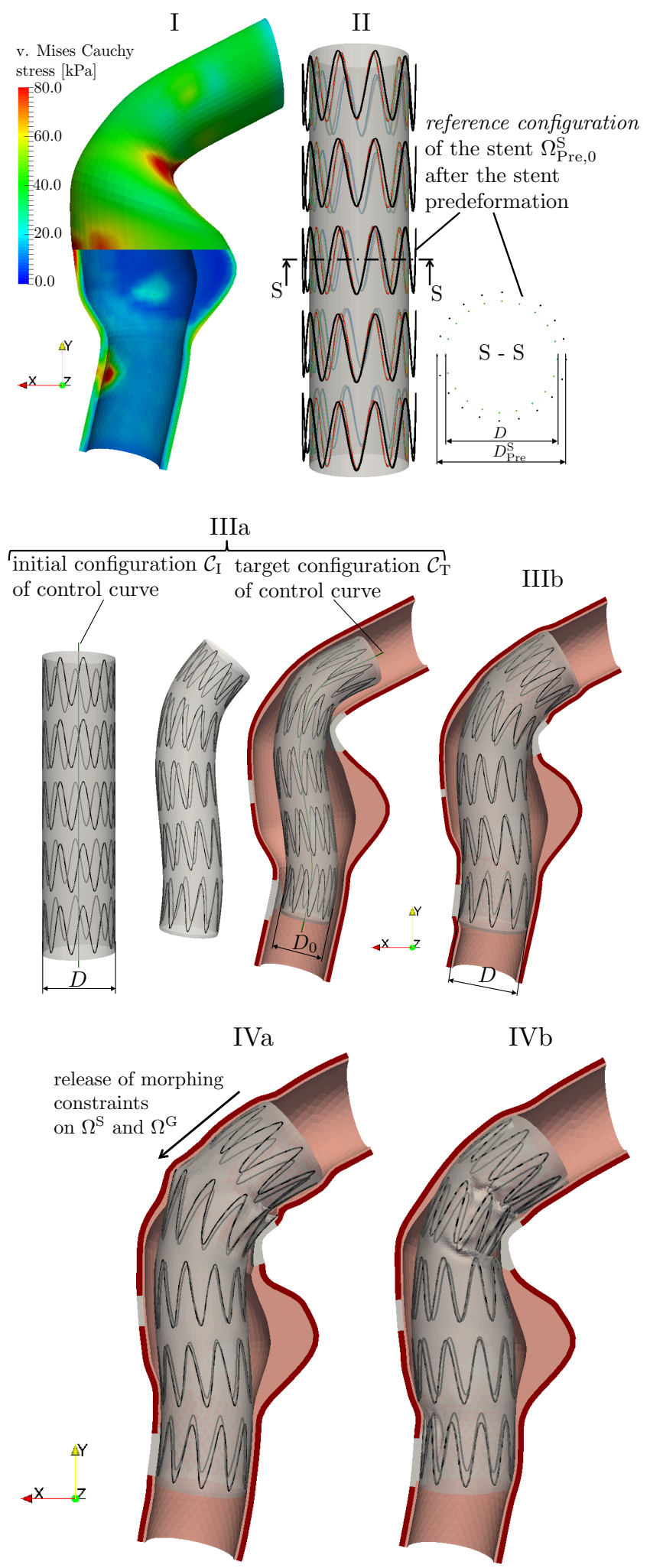

Fig. 5 Overview of the four steps of the in silico EVAR methodology: Vessel prestressing (I), stent predeformation (II), SG placement (IIIa+IIIb) and SG deployment $(\mathrm{IVa}+\mathrm{IVb})$. 
graft (equation (1e)). Application of the inverse morphing map $\tilde{\boldsymbol{M}}_{\text {Pre }}^{-1}$ changes the reference configuration of the stent (not the graft!) from $\Omega_{\mathrm{I}, 0}^{\mathrm{S}}, \boldsymbol{X}_{\mathrm{I}}^{\mathrm{S}} \in \Omega_{\mathrm{I}, 0}^{\mathrm{S}}$ (Figure $4 \mathrm{III})$ to $\Omega_{\mathrm{Pre}, 0}^{\mathrm{S}}, \boldsymbol{X}_{\mathrm{Pre}}^{\mathrm{S}} \in \Omega_{\mathrm{Pre}, 0}^{\mathrm{S}}$ (Figure $4 \mathrm{II}$ ) where $\tilde{\boldsymbol{M}}_{\text {Pre }}^{-1}$ is defined as

$$
\begin{gathered}
\tilde{\boldsymbol{M}}_{\text {Pre }}^{-1}\left(t_{\text {Pre }}(\xi), \Delta r_{\text {Pre }}(\xi), \mathrm{A}_{\text {Pre }}^{j}(\xi)\right): \\
\left\{\begin{array}{lll}
\left.\Omega_{\mathrm{I}, 0}^{\mathrm{S}}\right|_{\xi=0} & \rightarrow & \left.\Omega_{\text {Pre }, 0}^{\mathrm{S}}\right|_{\xi=1} \\
\left.\boldsymbol{X}_{\mathrm{I}}^{\mathrm{S}}\right|_{\xi=0} & \mapsto & \left.\boldsymbol{X}_{\text {Pre }}^{\mathrm{S}}\right|_{\xi=1}
\end{array},\right.
\end{gathered}
$$

$t_{\text {Pre }}(\xi)=t_{\mathrm{I}}$

$\Delta r_{\text {Pre }}(\xi)=\frac{1}{2} \xi\left(D_{\text {Pre }}^{\mathrm{S}}-D\right)$,

$\mathrm{A}_{\text {Pre }}^{j}(\xi)=\mathrm{A}_{\mathrm{I}}^{j}, \quad \forall j=1,2, \ldots, n_{\mathrm{C}}$.

Inserting equation (35b) into the linear path interpolation of equation (15) states that at any stage $\xi$ of the morphing map $\tilde{M}_{\text {Pre }}^{-1}$, the configuration of the control curve $\mathcal{C}$ is equal to the initial configuration of the control curve $\mathcal{C}_{\mathrm{I}}$. This means no control curve induced deformation of the morphing object takes place. The deformation is a pure change in the radius of the reference configuration of the stent from $D / 2$ to $D_{\text {Pre }}^{\mathrm{S}} / 2$ (equations (31) and (35c)) such that after the application of the map $\tilde{\boldsymbol{M}}_{\text {Pre }}^{-1}$ the new reference coordinates of the stent are given by $\boldsymbol{X}_{\mathrm{Pre}}^{\mathrm{S}}$. The reference configuration of the graft remains unchanged during this step, i.e. $\boldsymbol{X}_{\mathrm{Pre}}^{\mathrm{G}}=\boldsymbol{X}_{\mathrm{I}}^{\mathrm{G}}$ and $\Omega_{\mathrm{Pre}, 0}^{\mathrm{G}}=\Omega_{\mathrm{I}, 0}^{\mathrm{G}}$. All nodes of the stent are morphed as the morphing sets $A_{\text {Pre }}^{j}$ are constant and equal to the sets $A_{I}^{j}$ that correspond to the assignment based on the initial configuration $\mathcal{C}_{\mathrm{I}}$ of the control curve (cf. section 2.3.1).

As the morphing map $\tilde{\boldsymbol{M}}_{\text {Pre }}^{-1}$ changes the reference configuration from $\Omega_{\mathrm{I}, 0}^{\mathrm{S}}$ to $\Omega_{\mathrm{Pre}, 0}^{\mathrm{S}}$, this morphing map also affects the deformation map $\boldsymbol{\Phi}$ according to

$\Phi_{\text {Pre }}=\boldsymbol{\Phi} \circ \tilde{M}_{\text {Pre }}$

After application of the morphing map $\tilde{\boldsymbol{M}}_{\text {Pre }}^{-1}$, i.e. after application of the stent predeformation, the current configuration of the SG is related to the reference configuration via the deformation map (Figure 4)

$\boldsymbol{\Phi}_{\text {Pre }}\left(\boldsymbol{X}_{\text {Pre }}^{\{\mathrm{S}, \mathrm{G}\}}\right)=\boldsymbol{x}_{\text {Pre }}^{\{\mathrm{S}, \mathrm{G}\}}$,

where $\Omega_{\text {Pre }}^{\{\mathrm{S}, \mathrm{G}\}}, \boldsymbol{x}_{\text {Pre }}^{\{\mathrm{S}, \mathrm{G}\}} \in \Omega_{\mathrm{Pre}}^{\{\mathrm{S}, \mathrm{G}\}}$ are the current configurations of stent and graft after the stent predeformation. The change in the reference configuration of the stent from $\Omega_{\mathrm{I}, 0}^{\mathrm{S}}$ to $\Omega_{\mathrm{Pre}, 0}^{\mathrm{S}}$ in combination with the mesh tying constraint between stent and graft results in residual strains and stresses within the predeformed SG (cf. section 3.2.2). This approach for stent predeformation is valid for all types of SGs, exterior and interior stents and for all sizes of SGs.

\subsubsection{SG placement}

The in silico placement is a sequence of two steps (Figure $5 \mathrm{III} \mathrm{a}+\mathrm{b})$. The first step is a transformation of the SG from the initial, straight configuration $\Omega_{\mathrm{I}}^{\{\mathrm{S}, \mathrm{G}\}}$ onto the vessel centerline with a reduced SG diameter of $D_{0}$ such that the SG entirely fits into the vessel (Figure 5IIIa). A subsequent re-enlargement of the SG to the nominal diameter $D$ establishes potential contact between SG and vessel (Figure 5IIIb) which is modeled by the frictional contact constraints (1f)-(1g) with a friction coefficient of $\mu=0.4[36,64]$.

Both steps of the in silico placement are described by the morphing map $\tilde{\boldsymbol{m}}_{\mathrm{Pl}}$ which changes the current configuration of stent and graft from $\Omega_{\mathrm{I}}^{\{\mathrm{S}, \mathrm{G}\}}$, $\boldsymbol{x}_{\mathrm{I}}^{\{\mathrm{S}, \mathrm{G}\}} \in \Omega_{\mathrm{I}}^{\{\mathrm{S}, \mathrm{G}\}}$ (Figure 4III) to $\Omega_{\mathrm{Pl}}^{\{\mathrm{S}, \mathrm{G}\}}, \boldsymbol{x}_{\mathrm{Pl}}^{\{\mathrm{S}, \mathrm{G}\}} \in \Omega_{\mathrm{Pl}}^{\{\mathrm{S}, \mathrm{G}\}}$ (Figure 4IV) according to

$$
\begin{aligned}
& \tilde{\boldsymbol{m}}_{\mathrm{Pl}}\left(t_{\mathrm{Pl}}(\xi), \Delta r_{\mathrm{Pl}}(\xi), \mathrm{A}_{\mathrm{Pl}}^{j}(\xi)\right): \\
& \left\{\begin{array}{lll}
\left.\Omega_{\mathrm{I}}^{\{\mathrm{S}, \mathrm{G}\}}\right|_{\xi=0} & \rightarrow & \left.\Omega_{\mathrm{Pl}}^{\{\mathrm{S}, \mathrm{G}\}}\right|_{\xi=1} \\
\left.\boldsymbol{x}_{\mathrm{I}}^{\{\mathrm{S}, \mathrm{G}\}}\right|_{\xi=0} & \mapsto & \left.\boldsymbol{x}_{\mathrm{Pl}}^{\{\mathrm{S}, \mathrm{G}\}}\right|_{\xi=1}
\end{array},\right. \\
& t_{\mathrm{Pl}}(\xi)=\left\{\begin{array}{ll}
t_{\mathrm{I}}+2 \xi\left(t_{\mathrm{T}}-t_{\mathrm{I}}\right), & \xi<\frac{1}{2} \\
t_{\mathrm{T}}, & \xi \geq \frac{1}{2}
\end{array},\right. \\
& \Delta r_{\mathrm{Pl}}(\xi)=\left\{\begin{array}{ll}
-\xi\left(D-D_{0}\right), & \xi<\frac{1}{2} \\
(\xi-1)\left(D-D_{0}\right), & \xi \geq \frac{1}{2}
\end{array},\right. \\
& \mathrm{A}_{\mathrm{Pl}}^{j}(\xi)=\mathrm{A}_{\mathrm{I}}^{j}, \quad \forall j=1,2, \ldots, n_{\mathrm{C}} .
\end{aligned}
$$

Equation (38b) triggers a control curve induced deformation of the current configuration of the SG from the initial configuration of the control curve $\mathcal{C}_{\mathrm{I}}$ (centerline of the SG configuration $\Omega_{\mathrm{I}, 0}^{\{\mathrm{S}, \mathrm{G}\}}$ ) to the target configuration of the control curve $\mathcal{C}_{\mathrm{T}}$ (centerline of the vessel configuration $\left.\Omega_{\mathrm{I}, 0}^{\mathrm{Ao}}\right)$ for $\xi<\frac{1}{2}$. For $\xi \geq \frac{1}{2}$, no further control curve induced deformation takes place. The configuration of the control curve $\mathcal{C}(t(\xi))$ for $\xi \geq \frac{1}{2}$ is equal to the target configuration $\mathcal{C}_{\mathrm{T}}$ of the control curve. Equation (38c) states a change in the radius of stent and graft from $D / 2$ to $D_{0} / 2$ for $\xi<\frac{1}{2}$ and a change in the radius from $D_{0} / 2$ to $D / 2$ for $\xi \geq \frac{1}{2}$.

According to equation (38d) all nodes of the SG are morphed for any $\xi \in[0 ; 1]$. Hence, during the total in silico $\mathrm{SG}$ placement step, the current configuration of the entire $\mathrm{SG}$ is prescribed by the morphing map $\tilde{\boldsymbol{m}}_{\mathrm{Pl}}(\xi)$. Therefore, buckling of the SG is prevented 
during the whole placement process. Further, since the deformation of the entire $\mathrm{SG}$ is prescribed, the contact between SG and vessel reduces to a computationally simple Signorini contact problem. Still, the BVP of equation (1a)-(1g) has to be solved for the vessel $\Omega_{0}^{\text {Ao }}$. Those aspects make the in silico placement computationally very efficient and robust.

\subsubsection{SG deployment}

Before the SG is deployed, we apply the systolic pressure state $\hat{\boldsymbol{t}}^{\mathrm{Ao}}=\hat{\boldsymbol{t}}^{\mathrm{G}}=-p_{\mathrm{s}} \cdot \boldsymbol{n}$ on $\left(\gamma_{1, \mathrm{n}}^{\mathrm{Ao}} \cup \gamma_{\mathrm{l}}^{\mathrm{G}}\right)$ with $p_{\mathrm{s}}=130 \mathrm{mmHg}$ and remove the internal vessel pressure on the luminal vessel surface $\gamma_{1, c}^{\text {Ao }}$ covered by the SG, i.e. $\hat{\boldsymbol{t}}^{\mathrm{Ao}}=\mathbf{0}$ on $\gamma_{\mathrm{l}, \mathrm{c}}^{\mathrm{Ao}}($ Figure 1$)$.

During the SG deployment, the morphing constraints on the SG are gradually removed starting from the proximal end (Figure 5IVa) by application of the map

$$
\begin{aligned}
& \tilde{\boldsymbol{m}}_{\mathrm{De}}\left(t_{\mathrm{De}}(\xi), \Delta r_{\mathrm{De}}(\xi), \mathrm{A}_{\mathrm{De}}^{j}(\xi)\right) \text { : } \\
& \left\{\begin{array}{lll}
\left.\Omega_{\mathrm{Pl}}^{\{\mathrm{S}, \mathrm{G}\}}\right|_{\xi=0} & \rightarrow & \left.\Omega_{\mathrm{De}}^{\{\mathrm{S}, \mathrm{G}\}}\right|_{\xi=1} \\
\left.\boldsymbol{x}_{\mathrm{Pl}}^{\{\mathrm{S}, \mathrm{G}\}}\right|_{\xi=0} & \mapsto & \left.\boldsymbol{x}_{\mathrm{De}}^{\{\mathrm{S}, \mathrm{G}\}}\right|_{\xi=1}
\end{array},\right. \\
& t_{\mathrm{De}}(\xi)=t_{\mathrm{T}}, \\
& \Delta r_{\text {De }}(\xi)=0 \text {, } \\
& \mathrm{A}_{\mathrm{De}}^{j}(\xi)=\left\{\begin{array}{ll}
\mathrm{A}_{\mathrm{I}}^{j}, & \xi<\frac{j}{n_{\mathrm{C}}} \\
\varnothing, & \xi \geq \frac{j}{n_{\mathrm{C}}}
\end{array} \quad \forall j=1,2, \ldots, n_{\mathrm{C}},\right.
\end{aligned}
$$

where $\Omega_{\mathrm{Pl}}^{\{\mathrm{S}, \mathrm{G}\}}, \boldsymbol{x}_{\mathrm{Pl}}^{\{\mathrm{S}, \mathrm{G}\}} \in \Omega_{\mathrm{Pl}}^{\{\mathrm{S}, \mathrm{G}\}}$ (Figure 4IV) are the current configurations of stent and graft before the in silico $\mathrm{SG}$ deployment $(\xi=0)$ and $\Omega_{\mathrm{De}}^{\{\mathrm{S}, \mathrm{G}\}}$, $\boldsymbol{x}_{\mathrm{De}}^{\{\mathrm{S}, \mathrm{G}\}} \in \Omega_{\mathrm{De}}^{\{\mathrm{S}, \mathrm{G}\}}$ (Figure $4 \mathrm{~V}$ ) are the current configurations of stent and graft after the in silico deployment $(\xi=1)$. No control curve induced deformation and no morphing induced change in the radius (equation $(39 \mathrm{c}))$ takes place during the in silico SG deployment. The configuration of the control curve $\mathcal{C}\left(t_{\mathrm{De}}(\xi)\right)$ for $\xi \in[0 ; 1]$ is equal to the target configuration $\mathcal{C}_{\mathrm{T}}$ of the control curve (equation (39b)).

Equation $(39 \mathrm{~d})$ induces a gradual release of the morphing constraints from the nodes of the SG by gradual emptying of the morphing sets $\mathrm{A}_{\text {De }}^{j}(\xi)$. Only nodes that are part of the total morphing set $\mathrm{A}_{\mathrm{De}}$ (equation (32)) are prescribed by morphing constraints. As

$\mathrm{A}_{\mathrm{De}}(\xi=1)=\bigcup_{j=1}^{n_{\mathrm{C}}} \mathrm{A}_{\mathrm{De}}^{j}(\xi=1)=\varnothing$

for $\xi=1$, in the deployed state all morphing constraints are removed. Consequently, the current configurations $\Omega_{\mathrm{De}}^{(\Pi)}$ are related to the corresponding stress- free reference configurations $\Omega_{\mathrm{Pre}, 0}^{(\Pi)}$ solely by means of the deformation map

$$
\begin{aligned}
\boldsymbol{\Phi}_{\text {Pre }}\left(\boldsymbol{X}_{\text {Pre }}^{(\Pi)}\right) & =\left(\boldsymbol{\Phi} \circ \tilde{\boldsymbol{M}}_{\text {Pre }}\right)\left(\boldsymbol{X}_{\text {Pre }}^{(\Pi)}\right) \\
& =\boldsymbol{\Phi}(\underbrace{\tilde{\boldsymbol{M}}_{\mathrm{Pre}}\left(\boldsymbol{X}_{\text {Pre }}^{(\Pi)}\right)}_{=\boldsymbol{X}_{\mathrm{I}}^{(\Pi)}}) \\
& =\boldsymbol{x}_{\mathrm{De}}^{(\Pi)}
\end{aligned}
$$

(cf. Figure 4) with $\Pi=\{$ Ao, G, S $\}$ based on the BVP of equation (1a)-(1g). This means that the SG can elastically deform within the elastically deformable vessel. The final state of SG and vessel after the in silico deployment is visualized in Figure 5IVb.

\subsubsection{SG parameter continuation}

Correct sizing of the SG is one of the major challenges in the pre-operative planning phase of EVAR as the optimal diameter of the SG depends on various factors such as the shape of the vessel or the physical condition of the vessel. In classical studies investigating this issue $[69,16]$, for each considered SG diameter a separate in silico EVAR simulation was required. This can be computationally very expensive if many different SG diameters are to be studied. Therefore, here we propose a parameter continuation approach to model different SG diameters within one simulation.

After the in silico placement and deployment of a SG with the nominal diameter $D$, the nominal diameter of the SG can be modified continuously from $D$ to $D_{\mathrm{PC}}^{\mathrm{G}}$. Hence, all degrees of $\mathrm{SG}$ oversizing in the range $\left[\frac{D}{D^{\mathrm{Ao}}} ; \frac{D_{\mathrm{PC}}^{\mathrm{G}}}{D^{\mathrm{Ao}}}\right]$ can be investigated where $D^{\mathrm{Ao}}$ is the luminal diameter of the vessel in the landing zone.

The continuous change in the nominal diameter of the $\mathrm{SG}$ is realized by altering the reference configuration of the SG from $\Omega_{\mathrm{Pre}, 0}^{\{\mathrm{S}, \mathrm{G}\}}, \boldsymbol{X}_{\mathrm{Pre}}^{\{\mathrm{S}, \mathrm{G}\}} \in \Omega_{\mathrm{Pre}, 0}^{\{\mathrm{S}, \mathrm{G}\}}$ (Figure 4II) to $\Omega_{\mathrm{PC}, 0}^{\{\mathrm{S}, \mathrm{G}\}}, \boldsymbol{X}_{\mathrm{PC}}^{\{\mathrm{S}, \mathrm{G}\}} \in \Omega_{\mathrm{PC}, 0}^{\{\mathrm{S}, \mathrm{G}\}}$ (Figure $4 \mathrm{I}$ ) according to the morphing map

$$
\begin{array}{r}
\tilde{\boldsymbol{M}}_{\mathrm{PC}}^{-1}\left(t_{\mathrm{PC}}(\xi), \Delta r_{\mathrm{PC}}^{\mathrm{S}}(\xi), \Delta r_{\mathrm{PC}}^{\mathrm{G}}(\xi), \mathrm{A}_{\mathrm{PC}}^{j}\right): \\
\left\{\begin{array}{lll}
\left.\Omega_{\mathrm{Pre}, 0}^{\{\mathrm{S}, \mathrm{G}\}}\right|_{\xi=0} & \rightarrow & \left.\Omega_{\mathrm{PC}, 0}^{\{\mathrm{S}, \mathrm{G}\}}\right|_{\xi=1} \\
\left.\boldsymbol{X}_{\mathrm{Pre}}^{\{\mathrm{S}, \mathrm{G}\}}\right|_{\xi=0} & \mapsto & \left.\boldsymbol{X}_{\mathrm{PC}}^{\{\mathrm{S}, \mathrm{G}\}}\right|_{\xi=1},
\end{array}\right.
\end{array}
$$

$t_{\mathrm{PC}}(\xi)=t_{\mathrm{I}}$,

$\Delta r_{\mathrm{PC}}^{\mathrm{S}}(\xi)=\frac{1}{2} \xi\left(D_{\mathrm{PC}}^{\mathrm{S}}-D_{\mathrm{Pre}}^{\mathrm{S}}\right)$,

$\Delta r_{\mathrm{PC}}^{\mathrm{G}}(\xi)=\frac{1}{2} \xi\left(D_{\mathrm{PC}}^{\mathrm{G}}-D\right)$,

$\mathrm{A}_{\mathrm{PC}}^{j}(\xi)=\mathrm{A}_{\mathrm{I}}^{j}, \quad \forall j=1,2, \ldots, n_{\mathrm{C}}$. 
The configuration of the control curve $\mathcal{C}\left(t_{\mathrm{PC}}(\xi)\right)=\mathcal{C}_{\mathrm{I}}$ does not change during the morphing process (equation (42b)). The morphing map $\tilde{\boldsymbol{M}}_{\mathrm{PC}}^{-1}$ is a pure change in the diameter of the stent from $D_{\mathrm{Pre}}^{\mathrm{S}}$ to $D_{\mathrm{PC}}^{\mathrm{S}}$ (equation (42c)) and the diameter of the graft from $D$ to $D_{\mathrm{PC}}^{\mathrm{G}}$ (equation $(42 \mathrm{~d})$ ). The diameter $D_{\mathrm{PC}}^{\mathrm{S}}$ of the stent reference configuration $\Omega_{\mathrm{PC}, 0}^{\mathrm{S}}$ after the parameter continuation is chosen such that the degree of stent predeformation

$\frac{D_{\mathrm{Pre}}^{\mathrm{S}}}{D}=\frac{D_{\mathrm{PC}}^{\mathrm{S}}}{D_{\mathrm{PC}}^{\mathrm{G}}}$

is kept constant during the entire parameter continuation.

After the change in the nominal diameter of the SG from $D$ to $D_{\mathrm{PC}}^{\mathrm{G}}$, the relation between the stressfree reference configuration $\Omega_{\mathrm{PC}, 0}^{\{\mathrm{S}, \mathrm{G}\}}$ (Figure $4 \mathrm{I}$ ) and the current configuration $\Omega_{\mathrm{PC}}^{\{\mathrm{S}, \mathrm{G}\}}$ (Figure 4VI) is determined by the deformation map $\boldsymbol{\Phi}_{\mathrm{PC}}$ where

$\boldsymbol{\Phi}_{\mathrm{PC}}=\boldsymbol{\Phi}_{\mathrm{Pre}} \circ \tilde{\boldsymbol{M}}_{\mathrm{PC}}=\boldsymbol{\Phi} \circ \tilde{\boldsymbol{M}}_{\mathrm{Pre}} \circ \tilde{\boldsymbol{M}}_{\mathrm{PC}}$.

Consequently,

$$
\begin{aligned}
\boldsymbol{\Phi}_{\mathrm{PC}}\left(\boldsymbol{X}_{\mathrm{PC}}^{(\Pi)}\right) & =\left(\boldsymbol{\Phi} \circ \tilde{\boldsymbol{M}}_{\mathrm{Pre}} \circ \tilde{\boldsymbol{M}}_{\mathrm{PC}}\right)\left(\boldsymbol{X}_{\mathrm{PC}}^{(\Pi)}\right) \\
& =\boldsymbol{\Phi}(\underbrace{\tilde{\boldsymbol{M}}_{\mathrm{Pre}}(\overbrace{\tilde{\boldsymbol{M}}_{\mathrm{PC}}\left(\boldsymbol{X}_{\mathrm{PC}}^{(\Pi)}\right)}^{=\boldsymbol{X}_{\mathrm{Pre}}^{(\Pi)}})}_{=\boldsymbol{X}_{\mathrm{I}}^{(\Pi)}}) \\
& =\boldsymbol{x}_{\mathrm{PC}}^{(\Pi)}
\end{aligned}
$$

with $\Pi=\{$ Ao, G, S $\}$ (cf. Figure 4 ).

\subsection{Discretization and solution technique}

change in We apply geometrically nonlinear FEM to numerically solve the BVP of section 2.2. The stent is discretized by linear, hexahedral elements with enhanced assumed strain (EAS) technology whereas the graft is meshed by hexahedral solid-shell elements [79] with EAS and assumed natural strain (ANS) technology to reduce locking phenomena. The ILT is meshed by linear, tetrahedral and pyramid elements. Pyramid elements are utilized to guarantee conforming meshes between ILT and the vessel wall which is meshed by linear, hexahedral elements (Figure 7) with F-bar-based element technology [19] to circumvent volumetric locking of the nearly incompressible material behavior of the vessel.

We apply mortar-based discretization methods [67, 68] for mesh tying and contact interfaces $\Gamma_{\mathrm{m}}$ and $\gamma_{\mathrm{ex}}^{\mathrm{G}}$, respectively. The contact constraint enforcement (equation (1f) and (1g)) is based on a penalty regularization which represents a good compromise between accuracy and efficiency. The penalty parameters are chosen sufficiently large to reduce the penetration of contact bodies to a minimum.

A parallel iterative GMRES method preconditioned using algebraic multigrid [38] is utilized to solve the large system of equations resulting from the linearization of the Newton-type solver.

\section{Results}

\subsection{D morphing example based on RMF}

To show the accuracy and robustness of the morphing algorithm based on control curves with RMF, we morph an exemplary straight tube demonstrator. The discretized tube demonstrator and its centerline (control curve $\mathcal{C}_{\mathrm{I}}$ ) are given at pseudo-time $t=t_{\mathrm{I}}=0.0$. Using the proposed morphing algorithm, the straight tube demonstrator is morphed to a highly bend configuration at $t=t_{\mathrm{T}}=1.0$ which is defined by the given control curve $\mathcal{C}_{\mathrm{T}}$ at $t=t_{\mathrm{T}}=1.0$ (Figure $6 \mathrm{I}$ ). The control curve $\mathcal{C}_{\mathrm{T}}$ in the target configuration $\left(t=t_{\mathrm{T}}=1.0\right)$ is arbitrarily orientated in the global coordinate system to show the general applicability of the morphing algorithm in $3 \mathrm{D}$. The resulting morphing process has to be smooth in pseudo-time $t \in\left[t_{\mathrm{I}} ; t_{\mathrm{T}}\right]=[0.0 ; 1.0]$ and space $s \in[0.0 ; 1.0]$ where $s$ is the arclength parameter of the control curve of constant length 1 . The deformation of the tube demonstrator is fully described by morphing constraints, i.e. the given problem is a pure geometrical problem and the BVP of section 2.2 does not have to be solved. The RMF property (equations (7a)-(7c) and (16a)-(16c)) of the local coordinate frames aligned to the control curve is investigated in detail as this property is essential to obtain a smooth morphing process.

The RMF property in pseudo-time $t$ is visualized in Figure 6I. Red color markers on the bottom of the tube at $t=0.0$ indicate the direction of the normal vectors of the locally defined coordinate frames. The red color markers transform smoothly in time without undesired torsion around the centerline of the tube. Without specification of the RMF in pseudo-time $t$ (equations (16a)-(16c)), the tube would arbitrarily rotate around its centerline when transforming in pseudotime $t$.

The RMF property in space is additionally visualized in Figure 6II where the arrows are the normal vectors $\boldsymbol{n}^{j}(t)$ of the local coordinate frames aligned to the control curve at any pseudo-time $t$. The normal vectors $\boldsymbol{n}^{j}(t)$ indicate the orientation of the local coordinate frames. Due to the RMF property in space $s \in[0.0 ; 1.0]$ 

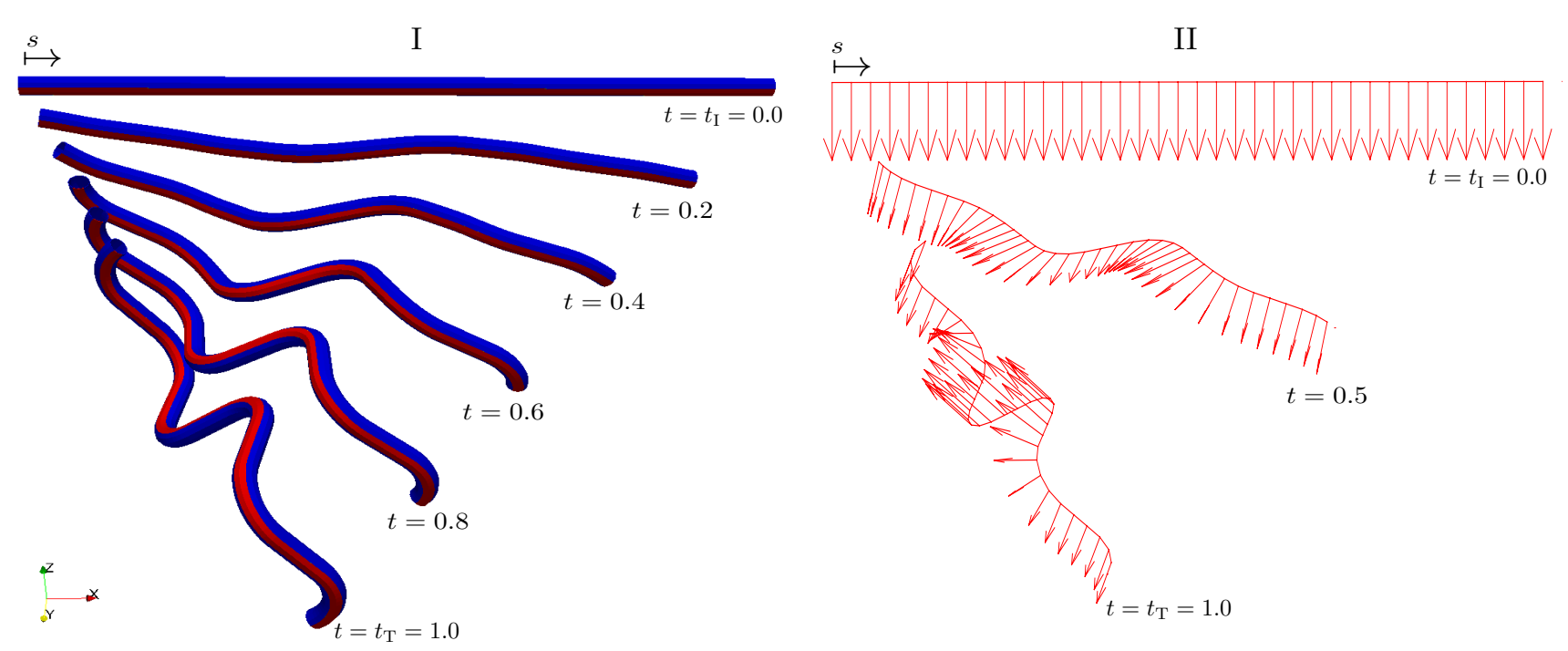

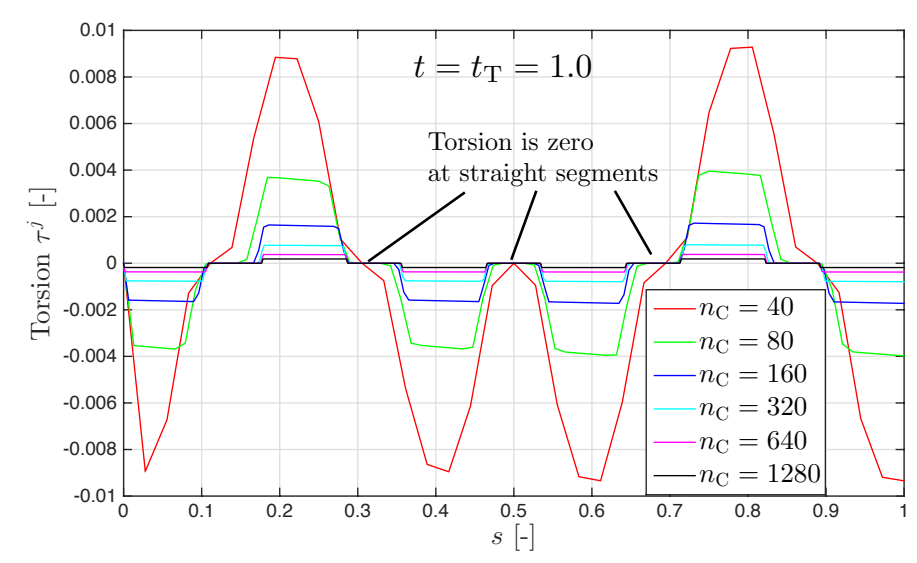

III

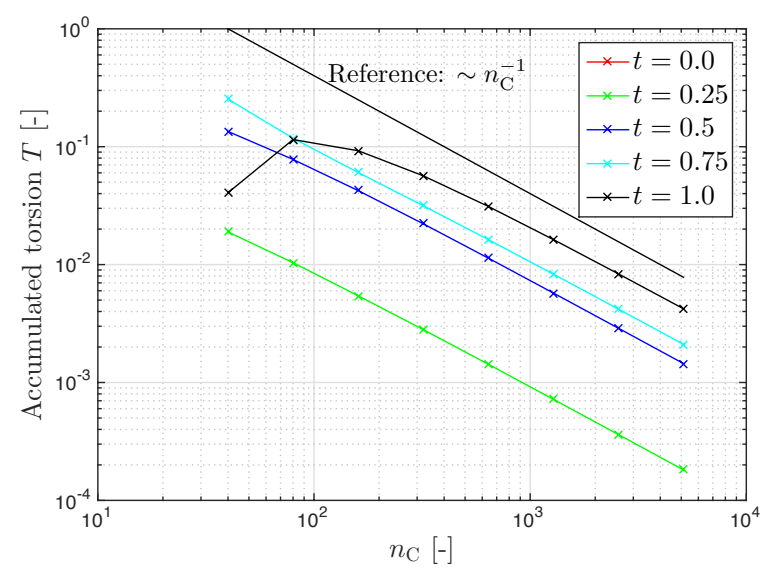

IV

Fig. 6 Morphing of an exemplary straight tube demonstrator to a highly bend tube: red markers indicate the direction of normal vectors of the locally defined coordinate frames (I); visualization of normal vectors of the locally defined coordinate frames according to the RMF definition (II); local (III) and accumulated (IV) convergence behavior of the RMF approximation for refinement of the control curve.

(equations (7a)-(7c)), the orientation of the normal vector $\boldsymbol{n}^{j}(t)$ varies smoothly along the spatial coordinate $s$. Any non-smooth variation of the orientation of the normal vectors along $s$ would result in an undesirable torsion $\tau^{j}$ of the morphing object (equation (6)).

In Figure 6III and 6IV, the convergence behavior of the suggested RMF approximation according to equations (7a)-(7c) is investigated for different discretizations of the control curve discretized by $n_{\mathrm{C}}$ points. By refinement of the control curve $\left(n_{\mathrm{C}} \rightarrow \infty\right)$, the local torsion converges to $\tau^{j}=0$ (Figure 6III, plotted for $t=1.0)$. The configuration $\mathcal{C}_{\mathrm{T}}=\mathcal{C}(t=1.0)$ of the control curve consists of five straight segments arbitrarily oriented in space (e.g. in the middle of the tube: $s=[0.47 ; 0.53])$. At these straight segments the RMF approximation scheme even leads to the exact solution of $\tau^{j}=0$ independent of the discretization of the curve with exception of $n_{\mathrm{C}}=40$ which discretization is too coarse to geometrically capture the straight segments. This property is essential for morphing SGs as SGs in the undeformed configuration naturally are straight.

Figure 6IV illustrates the convergence behavior of the accumulated torsion

$T=\sum_{j=1}^{n_{\mathrm{C}}}\left|\tau^{j}\right|\left\|\mathcal{C}^{\prime}\right\|$

for different pseudo-time steps $t$ which is a measure of the total angle of rotation of the normal vectors $\boldsymbol{n}^{j}(t)$ around curve $\mathcal{C}$. The convergence behavior is of first order independent of the considered pseudo-time step $t$. For $t=1.0$ coarse discretizations are not able to capture the full geometry of the curve as was already discussed for the local convergence behavior in figure 6III. The 
curve for $t=0.0$ is not visible in the logarithmic scale of figure $6 \mathrm{IV}$ as $\mathrm{T}=0$ for the perfectly straight curve.

\subsection{In silico EVAR in synthetic AAA geometry}

We demonstrate the approach of in silico EVAR with subsequent parameter continuation at the example of a tube SG with initial nominal diameter $D=25 \mathrm{~mm}$ (Figure 7II). Furthermore, the mechanical impact of stent predeformation of $15 \%$ with respect to the nominal diameter of the SG is examined. All other SG specific dimensions (e.g. thickness of stent wires and shape of stent limbs) are aligned to typical marketed SGs and are taken from [21]. The graft is a fabric with very little bending stiffness. In $[72,20,15]$ a reduced bending stiffness of the graft was enforced by reducing the graft thickness by a factor of 10 and increasing the elastic modulus by the same factor to retain the membrane stiffness of the graft. Here, we adopt this approach.

The SG is virtually deployed in a synthetic AAA geometry with moderate calcification and ILT in the aneurysm sac (Figure 7I). In this contribution we restrict the application of the in silico EVAR methodology to synthetic AAA geometries as these geometries have the advantage that geometrical parameters can easily be varied. In addition, the influence of certain parameters such as the SG oversize and the stent predeformation can be investigated more easily than in patient-specific geometries. In the considered synthetic AAA geometry, the neck angle of $60^{\circ}$ is chosen such that the vessel represents a critical candidate for EVAR. The instructions for use of the most marketed SGs are limited to neck angles below $60^{\circ}$ (e.g. [58]). Five calcification spots with non-constant $c^{\text {calc }}$-value are randomly placed within the synthetic AAA geometry. The size of the calcification spots is chosen such that $5 \%$ of the total volume of ILT and vessel wall are covered by calcifications (Figure 7I). The luminal neck and iliac diameter of the vessel are $22.5 \mathrm{~mm}$ resulting in a reasonable SG oversizing of approximately $10 \%$. A constant vessel wall thickness of $1.5 \mathrm{~mm}$ is used. The length of the proximal landing zone generally is limited by the position of the renal arteries which are not part of the vessel model. Nevertheless, the length of the proximal landing zone is assumed to be $20 \mathrm{~mm}$. The length of the distal landing zone is assumed to be $20 \mathrm{~mm}$ as well. Table 3 summarizes all geometrical parameters of SG and vessel.

A summary of the discretization of this example is given in table 4 and visualized in Figure 7.

The in silico EVAR according to the proposed methodology was performed on 56 cores (Intel Haswell nodes, SuperMUC, Leibniz Supercomputing Centre).

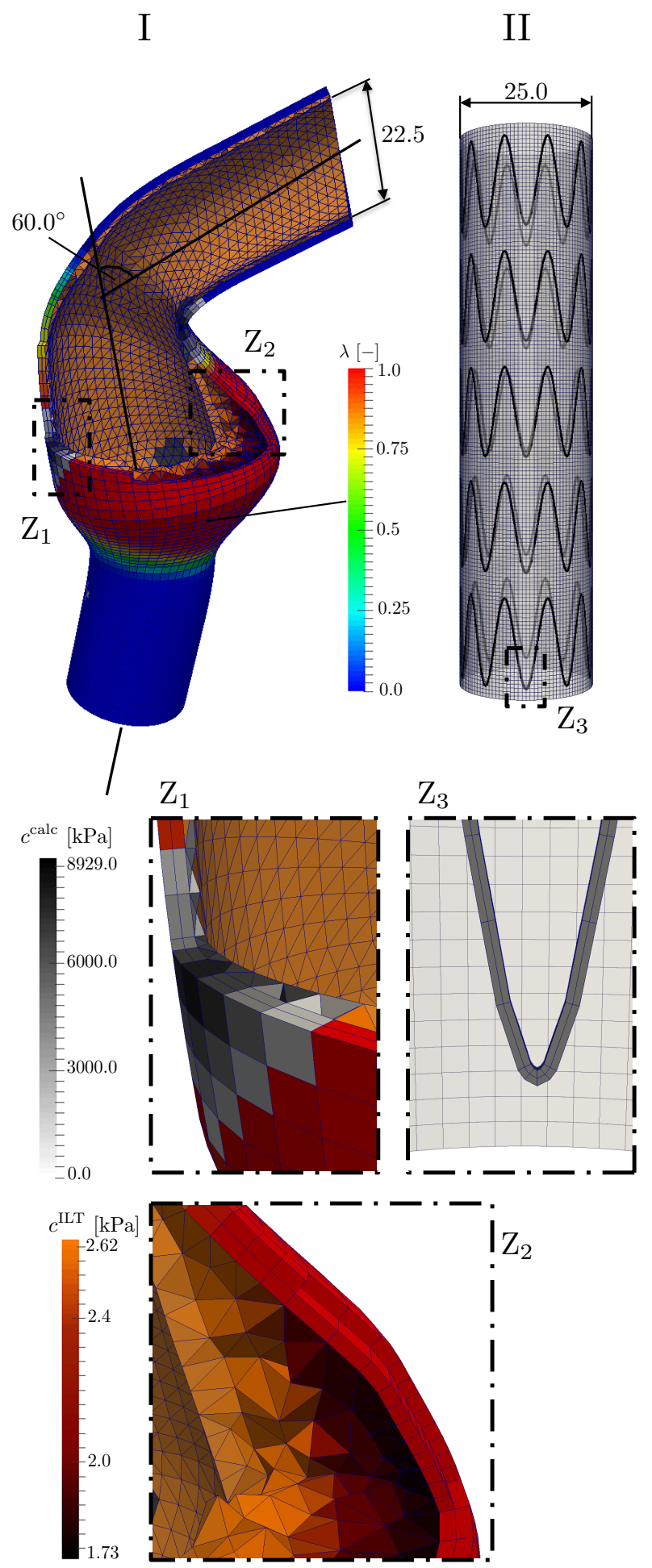

Fig. 7 Conforming mesh of a synthetic AAA (I) using a characteristic element length of $h^{\mathrm{Ao}}=1.0 \mathrm{~mm}$; mesh of the $\mathrm{SG}$ (II) using a characteristic element length of $h^{\mathrm{G}}=0.5 \mathrm{~mm}$ for the graft and an adaptive element size with mesh refinement in the curved parts for the stent. Visualization of the different constituents of the vessel and distribution of corresponding material parameters $c^{\text {calc }}, c^{\mathrm{ILT}}$ and $\lambda$. 
Table 3 Geometric parameters of vessel and SG.

\begin{tabular}{ll}
\hline \hline Vessel & \\
Luminal diameter proximal/iliac [mm] & 22.5 \\
Outer AAA diameter [mm] & 48.0 \\
Vessel wall thickness [mm] & 1.5 \\
Max thrombus thickness [mm] & 12.0 \\
Neck angle [-] & $60.0^{\circ}$ \\
Iliac angle [-] & $15.0^{\circ}$ \\
SG & \\
Nominal diameter D of SG [mm] & 25.0 \\
Stent diameter $D$ Pre $[\mathrm{mm}]$ & 28.75 \\
Length of SG [mm] & 102.5 \\
Graft thickness [mm] & 0.1 \\
Stent wire width/height [mm] & 0.33 \\
Stent limb height [mm] & 16.0 \\
Number of sinus periods per stent limb [-] & 10 \\
Number of stent limbs [-] & 5 \\
\hline \hline
\end{tabular}

Table 4 Summary of the number of degrees of freedom.

\begin{tabular}{lr|lr}
\hline \hline Stent: & 29,700 & Vessel wall: & 39,312 \\
Graft: & 98,784 & ILT/calc: & 17,676 \\
\hline$\sum$ SG: & 128,484 & $\sum$ vessel: & 56,988 \\
\hline \hline
\end{tabular}

The simulation took around $12 \mathrm{~h}$ and additional $8 \mathrm{~h}$ for the parameter continuation approach to vary the degree of SG oversizing from $10 \%$ to $30 \%$.

The results of the in silico EVAR for the SG with $10 \%$ oversizing are visualized in Figure 8 (left column). The four slices, which are all orthogonal to the vessel centerline, are selected such that $\mathrm{S}_{1}$ is a slice through the proximal landing zone, $\mathrm{S}_{2}$ is a slice in the region of maximum vessel curvature, $\mathrm{S}_{3}$ is a slice in the region of maximum AAA diameter and $\mathrm{S}_{4}$ is a slice through the distal landing zone.

In the proximal region of the vessel with a neck angle of $60^{\circ}$ the SG buckles in longitudinal direction. Consequently, the distribution of normal contact traction in the proximal landing zone is not uniform (Figure 8I). At the rather straight distal landing zone the distribution of contact tractions is more uniform. For the case of $10 \%$ SG oversizing no severe radial buckling of the $\mathrm{SG}$ is observed.

An important quantity is the normal passive fixation force of the SG

$\hat{F}=\int_{\gamma}\left\|\boldsymbol{t}_{\mathrm{n}}\right\| \mathrm{d} A$

which plays a key role in the sealing and prevention of type I endoleaks and SG migration [72]. We denote this force as passive fixation force as many SGs have barbs at the proximal end whose fixation force is denoted as active fixation force. $\boldsymbol{t}_{\mathrm{n}}$ is the normal contact traction at the contact interface between SG and luminal surface of the vessel. The outer surface of the SG covered by the most proximal stent limb and the outer surface of the SG covered by the most distal stent limb in the deformed, current configuration are chosen as integration areas $\gamma$ for the computation of the proximal fixation force $\hat{F}_{\text {prox }}$ and the distal fixation force $\hat{F}_{\text {dist }}$, respectively (Figure $8 \mathrm{I})^{3}$.

The von Mises Cauchy stresses of the vessel are maximal in the SG landing zones (green arrow) with its peak stresses in the calcified regions (red arrow) (Figure 8II). Furthermore, the insertion of the SG almost entirely removes the tissue stresses in the aneurysm sac as the SG with $10 \%$ oversizing is not in contact with the vessel in this region (Figure 8III, slice $\mathrm{S}_{3}$ ).

In Figure 8III, we consider the vessel maximum principal Green-Lagrange strains and the vessel deformation compared to the vessel configuration without SG insertion at the same pressure level of $p_{\mathrm{s}}=130 \mathrm{mmHg}$ (gray). In the strongly angulated neck the SG as well as the surrounding vessel have an elliptical shape (slice $\mathrm{S}_{2}$ ). Further, a reduction of the AAA diameter $\left(\right.$ slice $\mathrm{S}_{3}$ ) can be observed as the insertion of the $\mathrm{SG}$ fully removes the arterial pressure from the AAA sac.

\subsubsection{Examination of $S G$ oversizing using the parameter continuation approach}

Starting from the deployed configuration of the SG with $10 \%$ oversizing, the diameter of the $\mathrm{SG}$ is increased to a SG oversizing of $30 \%$ using the parameter continuation approach. According to equation (43), the ratio of the diameter change in the stent and the graft is identical such that the degree of stent predeformation remains constant during the increase of the SG oversizing. The change in the degree of SG oversizing from $10 \%$ to $30 \%$ leads to the following findings visualized in Figure 8 and 9:

- Passive normal fixation forces increase from $50.4 \mathrm{~N}$ to $77.0 \mathrm{~N}$ in the proximal landing zone and from $45.7 \mathrm{~N}$ to $76.8 \mathrm{~N}$ in the distal landing zone.

- Above $23 \%$ SG oversizing, the normal fixation force in the proximal landing zone does not further increase.

- Tissue strains and stresses increase almost linearly with increasing degree of SG oversizing.

- Radial buckling of the SG increases with increasing degree of SG oversizing (Figure 8III, blue arrows).

As the given problem is nonlinear, the results might be path-dependent. Especially, the parameter continuation approach has to be validated carefully with respect to this issue. Hence, to show that the use of the

3 The methodology can easily be extended to SG models with an uncovered stent in the proximal landing zone. However, such SG designs are not considered here for now. 

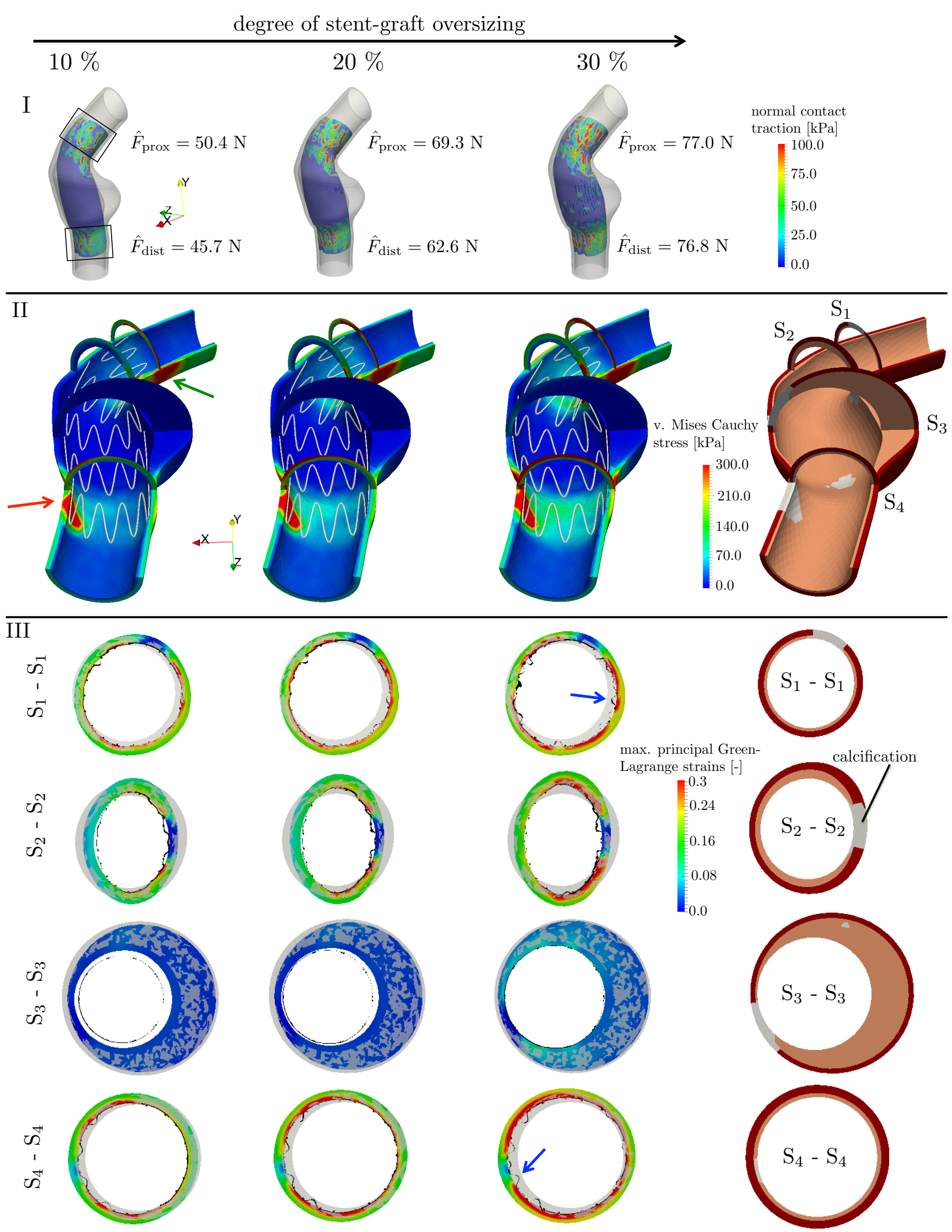

Fig. 8 Normal contact traction between SG and vessel (I), von Mises Cauchy stresses (II) and maximum principal GreenLagrange strains (III) in the vessel for different degrees of SG oversizing obtained by the SG parameter continuation approach; red arrow indicates peak stresses in the calcified regions and the green arrow maximum stresses in the non-calcified neck region; blue arrows highlight additional radial buckling of the graft due to increased SG oversizing; boxes in (I) indicate the proximal and distal landing zones used for the computation of the normal fixation forces. 


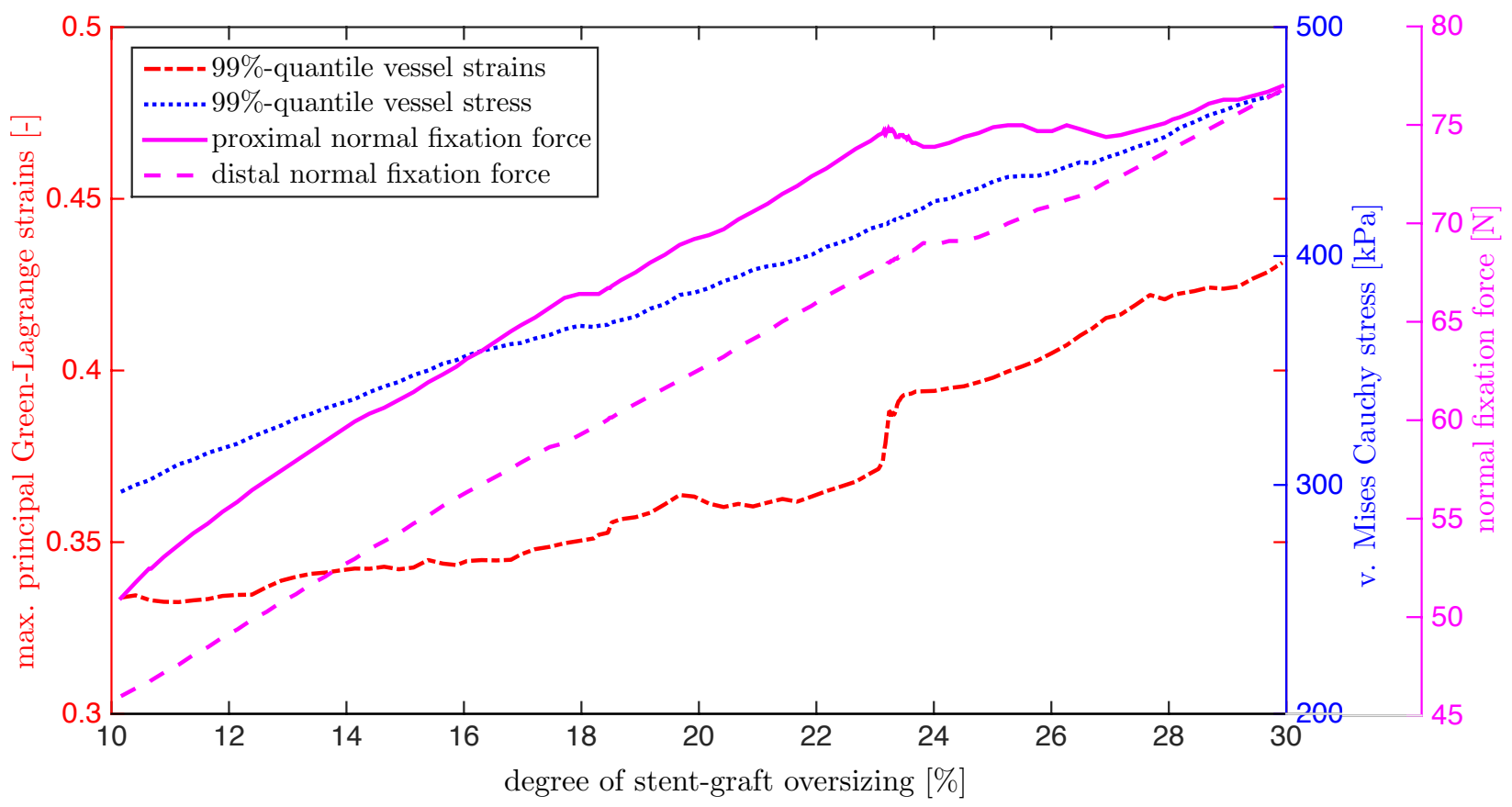

Fig. 9 Vessel maximum principal Green-Lagrange strains, vessel von Mises Cauchy stresses as well as SG normal fixation forces plotted over degree of SG oversizing for the example visualized in Figure 8.

parameter continuation approach for variable SG oversizing has a negligible influence on the final deployed state of the SG, we do the following comparison: we compare the results of the parameter continuation approach from $10 \%$ to $30 \%$ SG oversizing to the results of the direct approach for a SG oversized by $30 \%$ and the same synthetic AAA geometry as previously described. By the direct approach, we mean the proposed in silico EVAR methodology applied to a SG oversized by $30 \%$ (Figure 10).

Qualitatively only slight differences in the buckling pattern of the graft are visible (blue arrow) and marginal differences in the resulting stent diameters (Figure 10II). Quantitatively, we compare the absolute error $e=\bar{D}_{\text {cont }}^{\mathrm{S}}-\bar{D}_{\text {dir }}^{\mathrm{S}}$ between the resulting stent diameters of the two simulations where $\bar{D}_{\text {cont }}^{\mathrm{S}}$ and $\bar{D}_{\text {dir }}^{\mathrm{S}}$ are the stent diameters in the deployed state after the parameter continuation approach and the direct approach, respectively. This investigation shows an error with mean $\pm \mathrm{SD}$ of $\mu_{\mathrm{e}} \pm \sigma_{\mathrm{e}}=0.02 \pm 0.21 \mathrm{~mm}$.

\subsubsection{Examination of stent predeformation}

Most marketed SGs consist of stents with a diameter $D_{\text {Pre }}^{\mathrm{S}}>D$ where $D$ is the nominal diameter of the SG. In this section, we show the practicability of the approach for stent predeformation proposed in section 2.5.2. Further, we show the effect of stent prede- formation on passive fixation forces of the $\mathrm{SG}$, on the contact tractions between SG and vessel as well as on internal stress states of stent, graft and vessel.

First, we investigate the influence of stent predeformation on the internal stress states of stent and graft (Figure 11). In this example, the SG is supported by a very soft spring embedding on $\Gamma_{\mathrm{ex}}^{\mathrm{G}}$ to circumvent rigid body movement of the SG in the static analysis. The stiffness of the spring support is chosen as small as possible $(k=0.02 \mathrm{kPa} / \mathrm{mm})$ and was proofed to have negligible effects on the results. Starting from an initial configuration with stent diameter $D^{\mathrm{S}}=D$ and a mesh tying constraint between the outer stent surface $\Gamma_{\mathrm{ex}}^{\mathrm{S}}$ and the inner graft surface $\Gamma_{l}^{\mathrm{G}}$, we gradually change the stress-free reference configuration of the stent from $D$ to $D_{\text {Pre }}^{\mathrm{S}}=1.15 D$ by application of the morphing map $\tilde{\boldsymbol{M}}_{\text {Pre }}^{-1}$ according to equation (35a) (Figure 11I and 11II).

The change in the stress-free reference configuration of the stent in combination with the mesh tying constraint between stent and graft leads to residual strains and stresses within stent and graft. The maximum von Mises Cauchy stresses of $\sigma_{\text {Mises, } \max }^{\mathrm{S}}=360 \mathrm{MPa}$ occur in the curved regions of the stent. The residual stresses in the graft after the assembled SG with stent predeformation are very local at the regions connected to the stent. 

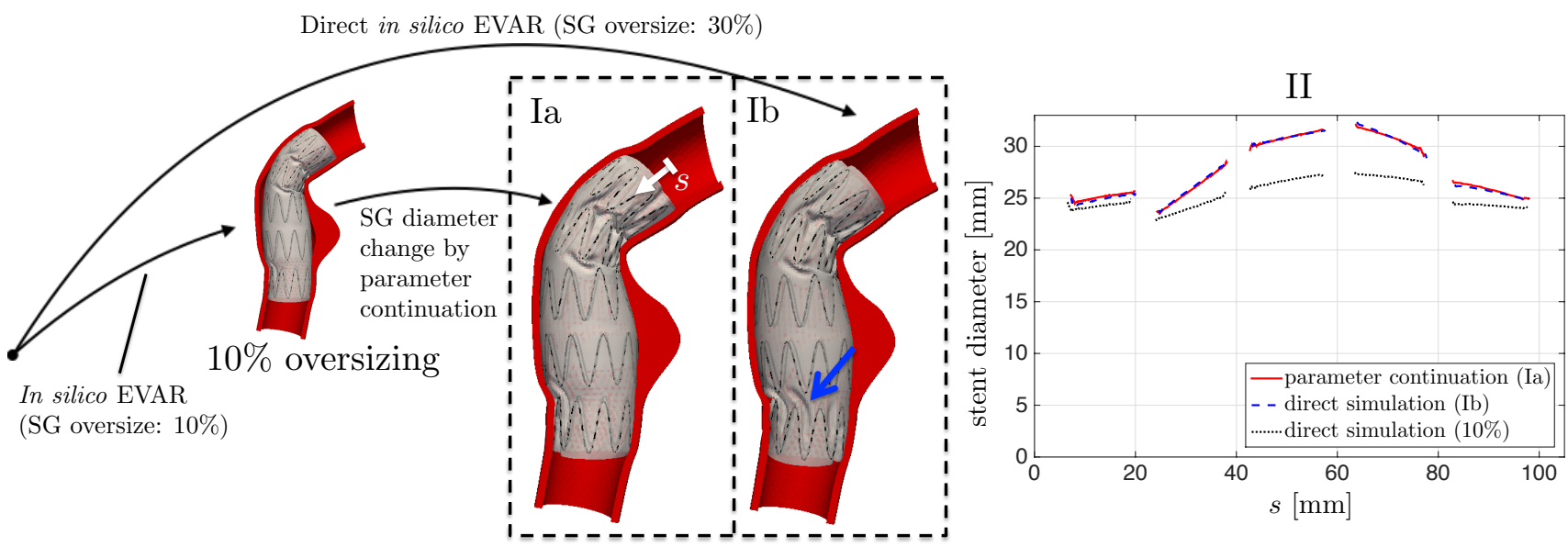

$30 \%$ oversizing

Fig. 10 Qualitative comparison of the results obtained by the in silico EVAR of a SG with 10\% oversizing and subsequent SG parameter continuation to a SG oversizing of $30 \%$ (Ia) with the results obtained by the in silico EVAR of a SG with $30 \%$ oversizing; blue arrow indicates marginal differences in the graft buckling. Quantitative comparison (II) of the stent diameter of the direct simulation (Ib) and the simulation based on the SG parameter continuation approach (Ia) plotted along the arclength $s$ of the centerline of the SG in the deployed state.

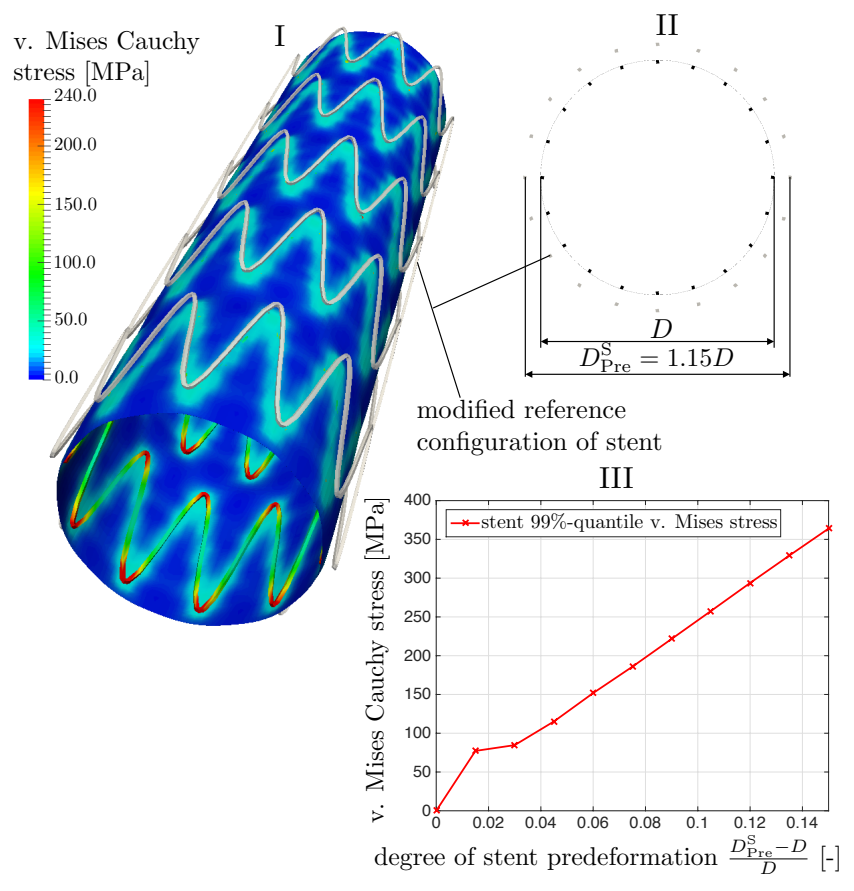

Fig. 11 Residual stresses after stent predeformation and stress-free reference configuration of the stent (gray) (I); nominal diameter $D$ of the SG and stent diameter $D_{\text {Pre }}^{\mathrm{S}}$ assumed for stent predeformation (II); relation between degree of stent predeformation and stent stresses (III).

Furthermore, in Figure 12 we investigate the influence of stent predeformation on SG and vessel in the deployed state for the SG oversized by $10 \%$. The comparison of the results of a SG with stent predeformation (Figure 12Ia+IIa) and the results of a SG without stent predeformation (Figure 12Ib+IIb) implies the following findings:

- Increased radial buckling of the graft in the angulated proximal neck in the case without stent predeformation. Only very little radial buckling in the case with stent predeformation.

- In the proximal landing zone the normal fixation force is $42 \%$ higher in the case with stent predeformation $\left(\hat{F}_{\text {prox }}=50.4 \mathrm{~N}\right)$ compared to the $\mathrm{SG}$ without stent predeformation $\left(\hat{F}_{\text {prox }}=35.5 \mathrm{~N}\right)$. In the distal landing zone the normal fixation force is $9 \%$ higher in the case with stent predeformation $\left(\hat{F}_{\text {dist }}=45.7 \mathrm{~N}\right)$ compared to the $\mathrm{SG}$ without stent predeformation $\left(\hat{F}_{\text {dist }}=42.0 \mathrm{~N}\right)$. (Figure $\left.12 \mathrm{I}\right)$

- This effect is also reflected in the stress state of the vessel: Stent predeformation leads up to $80 \mathrm{kPa}$ higher von Mises Cauchy stresses in the proximal and distal landing zones. (Figure 12II)

\section{Discussion}

It was shown that the proposed morphing algorithm based on control curves with RMF is a robust tool for in silico EVAR. The in silico EVAR methodology applied to a synthetic AAA geometry showed increased tissue strains and stresses in the proximal and distal landing zones. Wyss et al. [83] already claimed the relevance of the consideration of ILT and calcifications based on a statistical analysis of 217 patients. The in silico EVAR also showed distinct variations of local vessel stresses and strains related to the strong variations 

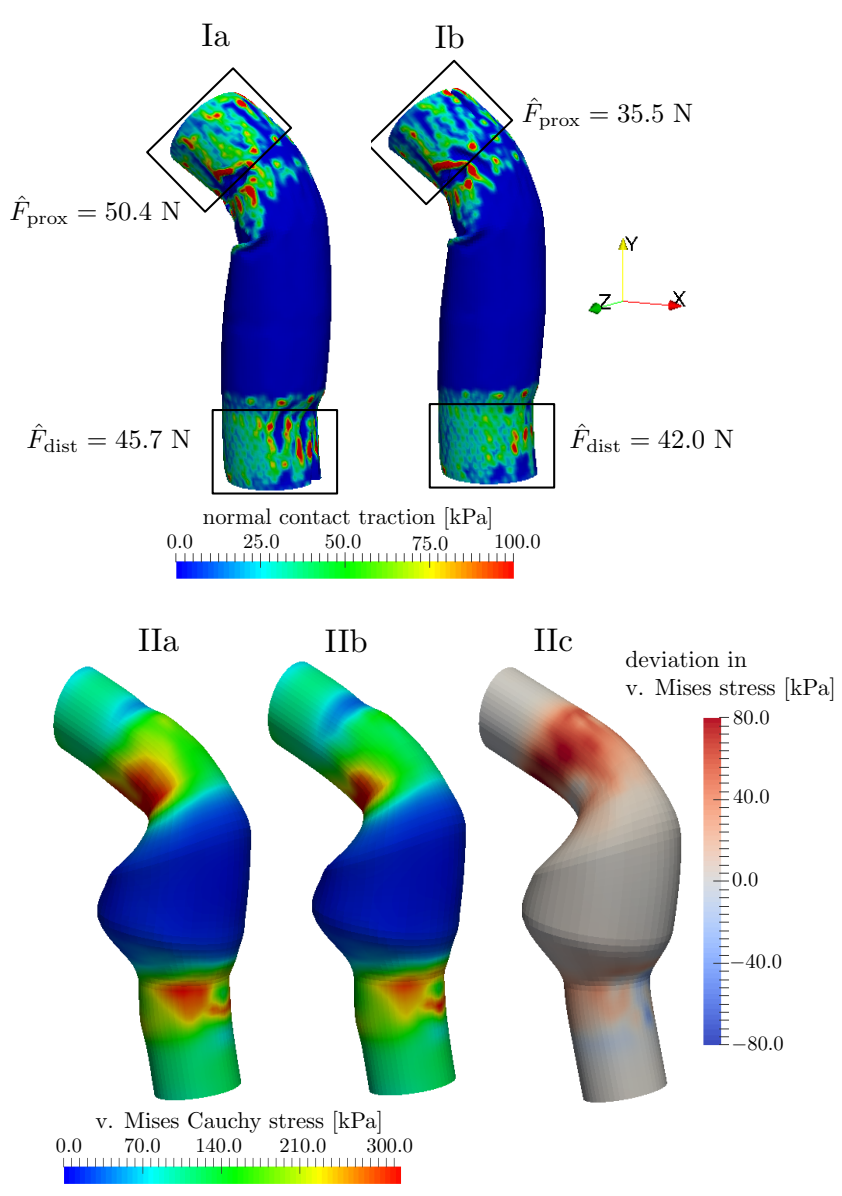

Fig. 12 Comparison of in silico EVAR results of a SG with $15 \%$ stent predeformation and a SG without stent predeformation $(0 \%)$ : comparison of normal contact tractions (Ia: $15 \%$ stent predeformation, Ib: $0 \%$ stent predeformation) and normal fixation forces $\hat{F}$; comparison of von Mises Cauchy stresses in the vessel (IIa: 15\% stent predeformation, IIb: 0\% stent predeformation, IIc: deviation between IIa and IIb)

of stiffness of the three vessel constituents ILT, calcifications and wall. The stiff behavior of calcifications has an influence on the expansion behavior of the SG in the landing zones which has to be further investigated in subsequent investigations. A reduction of the AAA diameter after the in silico EVAR could be observed due to the removal of the luminal pressure from the aneurysm sac. Similar observations, denoted as instantaneous change in AAA diameter, were reported e.g. in [23].

Small qualitative errors emerging from the pathdependency of the problem can be observed in the microscopic buckling pattern of the graft. However, the exact buckling pattern is not a point of interest when using the proposed methods of in silico EVAR and the parameter continuation approach for the investigation of SG oversizing. A reliable statement whether and how strong the graft radially buckles, which is stated to be an important indicator of EVAR complications $[75,16]$, is nevertheless possible. Considering the expansion diameter of the stent, the error using the parameter continuation approach for SG oversizing was shown to be negligibly small.

Investigations on the degree of SG oversizing using the parameter continuation approach showed almost linearly increasing passive normal fixation forces, vessel stresses and strains with increasing SG oversizing. However, the fixation forces in the strongly angulated neck, which are an important indicator of a proper SG fixation, did not further increase if the SG oversize was increased above $23 \%$. A possible explanation for this observation might be the increased radial buckling of the SG above $23 \%$ SG oversizing which has negative effects on the seal and force transmission between SG and vessel. However, as in this study only one exemplary vessel and one SG geometry were considered, these results do not allow for general conclusions. Further investigations on several vessel and SG geometries as well as comparisons to post-operative imaging are required.

It was shown that stent predeformation, whose major intention is the reduction of radial buckling of the graft, has an essential influence on the passive normal fixation forces of the SG and on vessel stresses. The influence of stent predeformation is larger in the strongly angulated proximal neck than in the rather straight distal landing zone as in the angulated neck the SG is rather prone to buckle. A linear relation between degree of stent predeformation and stresses in the stent struts can be observed which corresponds to the linear behavior between radial compression force and diameter reduction of SGs observed in [72].

\section{Limitations}

Apart from the model simplifications stated in section 2.1, this study is affected by five main limitations. First, the in silico EVAR methodology is a strongly simplified process compared to the real-world medical intervention. The in silico EVAR methodology does not model the single steps of the EVAR intervention itself but only aims at giving an approximation of the final deployed SG configuration under steady-state conditions.

Second, in this study the vessel wall was modeled with a constant wall thickness and we did not consider inter- or intra-patient variability of wall thickness and material parameters [7].

Third, large uncertainties arise in the frictional contact model and parameters. We modeled friction between SG and vessel based on Coulomb's law. A more 
elaborated frictional contact model should incorporate lubrication between vessel and SG which however cannot be considered by the proposed purely solid mechanical model.

Another limitation is the negligence of residual sac pressure after EVAR. In our model we assumed zero sac pressure after the insertion of the SG. However, in literature often a reduced but non-zero sac pressure in the AAA is reported $[13,49]$.

Finally, in this study the application of the in silico EVAR methodology was restricted to synthetic tube SGs. In future work the methodology has to be applied to patient-specific and bifurcated AAA geometries. Also, the accuracy of the methodology has to be validated using pre- and post-operative patient-specific data similar to the studies of Auricchio et al. [4] and Perrin et al. [64].

\section{Conclusions}

EVAR is a challenging intervention with still nonnegligible complication rates. This raises the need for better pre-operative planning tools. We have proposed a methodology for in silico EVAR intervention in AAAs which enables deeper insight into the mechanical state of the AAA and the SG after EVAR, such as contact forces and wall stresses. Special focus was put on advanced modeling of the vessel constituents ILT, calcifications, "healthy" vessel wall as well as "aneurysmatic" vessel wall.

A parameter continuation approach was applied for the first time to enable an efficient investigation of different degrees of SG oversizing. A novel methodology to consider stent predeformation was developed and it was shown that stent predeformation can have major effects on the mechanical state of SG and vessel after SG insertion.

In summary, the in silico EVAR methodology provides a robust and efficient approximation of the configurations of SG and vessel in the deployed state and hence is a first step towards a medical tool for a better risk assessment of the EVAR intervention as well as for a better SG selection and sizing.

Acknowledgements The authors gratefully acknowledge support and funding by the Leibniz Rechenzentrum München (LRZ) of the Bavarian Academy of Sciences under contract number pr48ta.

Conflict of Interest All authors declare that no conflicts of interest exist.

\section{References}

1. M. Alexa, D. Cohen-Or, and D. Levin. As-rigid-aspossible shape interpolation. In Proceedings of the 27th Annual Conference on Computer Graphics and Interactive Techniques, pages 157-164, New York, NY, USA, 2000. ACM Press/Addison-Wesley Publishing Co.

2. H.-E. Altnji, B. Bou-Saïd, and H. Walter-Le Berre. Morphological and stent design risk factors to prevent migration phenomena for a thoracic aneurysm: a numerical analysis. Medical engineering and physics, 37(1):23-33, 2015.

3. F. Auricchio, M. Conti, M. De Beule, G. De Santis, and B. Verhegghe. Carotid artery stenting simulation: from patient-specific images to finite element analysis. Medical engineering and physics, 33(3):281-289, 2011.

4. F. Auricchio, M. Conti, S. Marconi, A. Reali, J. L. Tolenaar, and S. Trimarchi. Patient-specific aortic endografting simulation: From diagnosis to prediction. Computers in Biology and Medicine, 43(4):386 - 394, 2013.

5. H. G. Beebe, J. L. Cronenwett, B. T. Katzen, D. C. Brewster, R. M. Green, V. E. T. Investigators, et al. Results of an aortic endograft trial: impact of device failure beyond 12 months. Journal of vascular surgery, 33(2):55$63,2001$.

6. T. Beier and S. Neely. Feature-based image metamorphosis. Computer Graphics, 26(2):35-42, 1992.

7. J. Biehler, M. W. Gee, and W. A. Wall. Towards efficient uncertainty quantification in complex and largescale biomechanical problems based on a bayesian multi-fidelity scheme. Biomechanics and modeling in mechanobiology, 14(3):489-513, 2015.

8. J. Bloomenthal. Calculation of reference frames along a space curve. Graphics gems, 1:567-571, 1990.

9. L. Canaud, P. Alric, M. Laurent, T.-P. Baum, P. Branchereau, C. H. Marty-Ané, and J.-P. Berthet. Proximal fixation of thoracic stent-grafts as a function of oversizing and increasing aortic arch angulation in human cadaveric aortas. Journal of Endovascular Therapy, 15(3):326-334, 2008.

10. T. E. Carew, R. N. Vaishnav, and D. J. Patel. Compressibility of the arterial wall. Circulation research, 23(1):6168, 1968.

11. E. Carmel and D. Cohen-Or. Warp-guided object-space morphing. The Visual Computer, 13(9):465-478, 1998.

12. R. W. Chang, P. Goodney, L.-Y. Tucker, S. Okuhn, H. Hua, A. Rhoades, N. Sivamurthy, and B. Hill. Tenyear results of endovascular abdominal aortic aneurysm repair from a large multicenter registry. Journal of vascular surgery, 58(2):324-332, 2013.

13. T. Chuter, K. Ivancev, M. Malina, T. Resch, J. Brunkwall, B. Lindblad, and B. Risberg. Aneurysm pressure following endovascular exclusion. European journal of vascular and endovascular surgery, 13(1):85-87, 1997.

14. F. Cochennec, J. Becquemin, P. Desgranges, E. Allaire, H. Kobeiter, and F. Roudot-Thoraval. Limb graft occlusion following evar: clinical pattern, outcomes and predictive factors of occurrence. European journal of vascular and endovascular surgery, 34(1):59-65, 2007.

15. S. De Bock, F. Iannaccone, M. De Beule, D. Van Loo, F. Vermassen, B. Verhegghe, and P. Segers. Filling the void: A coalescent numerical and experimental technique to determine aortic stent graft mechanics. Journal of biomechanics, 46(14):2477-2482, 2013.

16. S. De Bock, F. Iannaccone, M. De Beule, F. Vermassen, P. Segers, and B. Verhegghe. What if you stretch the 
ifu? a mechanical insight into stent graft instructions for use in angulated proximal aneurysm necks. Medical engineering and physics, 36(12):1567-1576, 2014.

17. S. De Bock, F. Iannaccone, G. De Santis, M. De Beule, P. Mortier, B. Verhegghe, and P. Segers. Our capricious vessels: the influence of stent design and vessel geometry on the mechanics of intracranial aneurysm stent deployment. Journal of biomechanics, 45(8):1353-1359, 2012.

18. S. De Bock, F. Iannaccone, G. De Santis, M. De Beule, D. Van Loo, D. Devos, F. Vermassen, P. Segers, and B. Verhegghe. Virtual evaluation of stent graft deployment: a validated modeling and simulation study. Journal of the mechanical behavior of biomedical materials, 13:129-139, 2012.

19. E. de Souza Neto, D. Perić, M. Dutko, and D. Owen. Design of simple low order finite elements for large strain analysis of nearly incompressible solids. International Journal of Solids and Structures, 33(20):32773296, 1996.

20. N. Demanget, S. Avril, P. Badel, L. Orgéas, C. Geindreau, J.-N. Albertini, and J.-P. Favre. Computational comparison of the bending behavior of aortic stent-grafts. Journal of the mechanical behavior of biomedical materials, 5(1):272-282, 2012.

21. N. Demanget, A. Duprey, P. Badel, L. Orgéas, S. Avril, C. Geindreau, J.-N. Albertini, and J.-P. Favre. Finite element analysis of the mechanical performances of 8 marketed aortic stent-grafts. Journal of Endovascular Therapy, 20(4):523-535, 2013.

22. S. Doll and K. Schweizerhof. On the development of volumetric strain energy functions. Journal of applied mechanics, 67(1):17-21, 2000.

23. S. H. Ellozy, A. Carroccio, R. A. Lookstein, T. S. Jacobs, M. D. Addis, V. J. Teodorescu, and M. L. Marin. Abdominal aortic aneurysm sac shrinkage after endovascular aneurysm repair: correlation with chronic sac pressure measurement. Journal of vascular surgery, 43(1):27, 2006.

24. R. T. Farouki. Exact rotation-minimizing frames for spatial pythagorean-hodograph curves. Graphical Models, 64(6):382-395, 2002.

25. M. F. Fillinger, S. P. Marra, M. L. Raghavan, and F. E. Kennedy. Prediction of rupture risk in abdominal aortic aneurysm during observation: wall stress versus diameter. Journal of vascular surgery, 37(4):724-732, 2003.

26. T. L. Forbes, G. DeRose, S. W. Kribs, and K. A. Harris. Cumulative sum failure analysis of the learning curve with endovascular abdominal aortic aneurysm repair. Journal of vascular surgery, 39(1):102-108, 2004.

27. T. C. Gasser, M. Auer, F. Labruto, J. Swedenborg, and J. Roy. Biomechanical rupture risk assessment of abdominal aortic aneurysms: model complexity versus predictability of finite element simulations. European Journal of Vascular and Endovascular Surgery, 40(2):176$185,2010$.

28. T. C. Gasser, G. Görgülü, M. Folkesson, and J. Swedenborg. Failure properties of intraluminal thrombus in abdominal aortic aneurysm under static and pulsating mechanical loads. Journal of vascular surgery, 48(1):179$188,2008$.

29. T. C. Gasser, R. W. Ogden, and G. A. Holzapfel. Hyperelastic modelling of arterial layers with distributed collagen fibre orientations. Journal of the royal society interface, 3(6):15-35, 2006

30. M. Gee, C. Förster, and W. Wall. A computational strategy for prestressing patient-specific biomechanical problems under finite deformation. International Journal for
Numerical Methods in Biomedical Engineering, 26(1):5272,2010 .

31. J. Gindre, A. Bel-Brunon, A. Kaladji, A. Duménil, M. Rochette, A. Lucas, P. Haigron, and A. Combescure. Finite element simulation of the insertion of guidewires during an evar procedure: example of a complex patient case, a first step toward patient-specific parameterized models. International journal for numerical methods in biomedical engineering, 31(7), 2015.

32. E. Goldstein and C. Gotsman. Polygon morphing using a multiresolution representation. In Graphics Interface, pages 247-247. Canadian Information Processing Society, 1995.

33. R. M. Greenhalgh, L. C. Brown, and J. T. Powell. Endovascular versus open repair of abdominal aortic aneurysm. New England Journal of Medicine, 362(20):1863-1871, 2010.

34. R. M. Greenhalgh and J. T. Powell. Endovascular repair of abdominal aortic aneurysm. New England Journal of Medicine, 358(5):494-501, 2008.

35. H. Guggenheimer. Computing frames along a trajectory. Computer Aided Geometric Design, 6(1):77-78, 1989.

36. G. J. Hall and E. P. Kasper. Comparison of element technologies for modeling stent expansion. Journal of Biomechanical Engineering, 128(5):751-756, 032006.

37. D. Haskett, G. Johnson, A. Zhou, U. Utzinger, and J. V. Geest. Microstructural and biomechanical alterations of the human aorta as a function of age and location. Biomechanics and modeling in mechanobiology, 9(6):725-736, 2010.

38. M. A. Heroux, R. A. Bartlett, V. E. Howle, R. J. Hoekstra, J. J. Hu, T. G. Kolda, R. B. Lehoucq, K. R. Long, R. P. Pawlowski, E. T. Phipps, et al. An overview of the trilinos project. ACM Transactions on Mathematical Software (TOMS), 31(3):397-423, 2005.

39. G. A. Holzapfel, M. Stadler, and T. C. Gasser. Changes in the mechanical environment of stenotic arteries during interaction with stents: computational assessment of parametric stent designs. Journal of biomechanical engineering, 127(1):166-180, 2005.

40. J. Humphrey and G. A. Holzapfel. Mechanics, mechanobiology, and modeling of human abdominal aorta and aneurysms. Journal of biomechanics, 45(5):805-814, 2012.

41. F. Iannaccone, M. De Beule, S. De Bock, I. M. Van der Bom, M. J. Gounis, A. K. Wakhloo, M. Boone, B. Verhegghe, and P. Segers. A finite element method to predict adverse events in intracranial stenting using microstents: In vitro verification and patient specific case study. Annals of biomedical engineering, 44(2):442-452, 2016.

42. W. D. Jordan, K. Ouriel, M. Mehta, D. Varnagy, W. M. Moore, F. R. Arko, J. Joye, J.-P. P. de Vries, J. P. de Vries, H. Eckstein, et al. Outcome-based anatomic criteria for defining the hostile aortic neck. Journal of vascular surgery, 61(6):1383-1390, 2015.

43. M. Kalteis, P. Benedikt, F. Huber, F. Haller, M. Kastner, and H. Lugmayr. Looking for a learning curve in evar based on the zenith stent graft. International Journal of Angiology, 21(04):223-228, 2012.

44. T. Kanai, H. Suzuki, and F. Kimura. 3d geometric metamorphosis based on harmonic map. In Computer Graphics and Applications, 1997. Proceedings., The Fifth Pacific Conference on, pages 97-104, 1997.

45. A. Katsargyris, D. Spinelli, K. Oikonomou, H. Mufty, and E. L. Verhoeven. Incomplete expansion of chimney stent graft during chimney-thoracic endovascular aneurysm repair. Annals of vascular surgery, 39:293-e1, 2017. 
46. C. Kleinstreuer, Z. Li, C. Basciano, S. Seelecke, and M. Farber. Computational mechanics of nitinol stent grafts. Journal of biomechanics, 41(11):2370-2378, 2008.

47. F. Klok. Two moving coordinate frames for sweeping along a 3d trajectory. Computer Aided Geometric Design, 3(3):217-229, 1986.

48. G. N. Kouvelos, K. Oikonomou, G. A. Antoniou, E. L. Verhoeven, and A. Katsargyris. A systematic review of proximal neck dilatation after endovascular repair for abdominal aortic aneurysm. Journal of Endovascular Therapy, 24(1):59-67, 2017.

49. S. Kwon, J. Rectenwald, and S. Baek. Intrasac pressure changes and vascular remodeling after endovascular repair of abdominal aortic aneurysms: review and biomechanical model simulation. Journal of biomechanical engineering, 133(1):011011, 2011.

50. F. Lazarus, S. Coquillart, and P. Jancene. Axial deformations: an intuitive deformation technique. ComputerAided Design, 26(8):607-613, 1994.

51. A. Lerios, C. D. Garfinkle, and M. Levoy. Feature-based volume metamorphosis. In Proceedings of the 22nd annual conference on Computer graphics and interactive techniques, pages 449-456. ACM, 1995.

52. K. K. Lin, J. A. Kratzberg, and M. L. Raghavan. Role of aortic stent graft oversizing and barb characteristics on folding. Journal of vascular surgery, 55(5):1401-1409, 2012.

53. A. Maier, M. Gee, C. Reeps, H.-H. Eckstein, and W. Wall. Impact of calcifications on patient-specific wall stress analysis of abdominal aortic aneurysms. Biomechanics and modeling in mechanobiology, 9(5):511-521, 2010.

54. A. Maier, M. W. Gee, C. Reeps, J. Pongratz, H.-H. Eckstein, and W. A. Wall. A comparison of diameter, wall stress, and rupture potential index for abdominal aortic aneurysm rupture risk prediction. Annals of biomedical engineering, 38(10):3124-3134, 2010.

55. G. Maleux, M. Koolen, and S. Heye. Complications after endovascular aneurysm repair. In Seminars in interventional radiology, volume 26, pages 3-9, 2009.

56. I. Mohan, R. Laheij, and P. Harris. Risk factors for endoleak and the evidence for stent-graft oversizing in patients undergoing endovascular aneurysm repair. European Journal of Vascular and Endovascular Surgery, 21(4):344-349, 2001.

57. P. Moireau, N. Xiao, M. Astorino, C. A. Figueroa, D. Chapelle, C. A. Taylor, and J.-F. Gerbeau. External tissue support and fluid-structure simulation in blood flows. Biomechanics and modeling in mechanobiology, 11(1-2):1-18, 2012.

58. F. L. Moll, J. Powell, G. Fraedrich, F. Verzini, S. Haulon, M. Waltham, J. Van Herwaarden, P. Holt, J. Van Keulen, B. Rantner, et al. Management of abdominal aortic aneurysms clinical practice guidelines of the european society for vascular surgery. European Journal of Vascular and Endovascular Surgery, 41:S1-S58, 2011.

59. S. Morlacchi, S. G. Colleoni, R. Cárdenes, C. Chiastra, J. L. Diez, I. Larrabide, and F. Migliavacca. Patientspecific simulations of stenting procedures in coronary bifurcations: two clinical cases. Medical engineering Éamp; physics, 35(9):1272-1281, 2013.

60. P. Mortier, G. A. Holzapfel, M. De Beule, D. Van Loo, Y. Taeymans, P. Segers, P. Verdonck, and B. Verhegghe. A novel simulation strategy for stent insertion and deployment in curved coronary bifurcations: comparison of three drug-eluting stents. Annals of biomedical engineering, 38(1):88-99, 2010.
61. J. A. Niestrawska, C. Viertler, P. Regitnig, T. U. Cohnert, G. Sommer, and G. A. Holzapfel. Microstructure and mechanics of healthy and aneurysmatic abdominal aortas: experimental analysis and modelling. Journal of The Royal Society Interface, 13(124):20160620, 2016.

62. S. Ockert, D. Boeckler, J. Allenberg, and H. Schumacher. Rupturiertes abdominelles aortenaneurysma. Gefaesschirurgie, 12(5):379-391, 2007.

63. R. Ogden. Large deformation isotropic elasticity-on the correlation of theory and experiment for incompressible rubberlike solids. In Proceedings of the Royal Society of London A: Mathematical, Physical and Engineering Sciences, volume 326, pages 565-584. The Royal Society, 1972.

64. D. Perrin, P. Badel, L. Orgéas, C. Geindreau, A. Dumenil, J.-N. Albertini, and S. Avril. Patient-specific numerical simulation of stent-graft deployment: Validation on three clinical cases. Journal of Biomechanics, 48(10):1868 - 1875, 2015.

65. D. Perrin, P. Badel, L. Orgeas, C. Geindreau, S. rolland du Roscoat, J.-N. Albertini, and S. Avril. Patientspecific simulation of endovascular repair surgery with tortuous aneurysms requiring flexible stent-grafts. Journal of the Mechanical Behavior of Biomedical Materials, 63:86-99, 2016.

66. D. Perrin, N. Demanget, P. Badel, S. Avril, L. Orgéas, C. Geindreau, and J.-N. Albertini. Deployment of stent grafts in curved aneurysmal arteries: toward a predictive numerical tool. International journal for numerical methods in biomedical engineering, 31(1), 2015.

67. A. Popp, M. W. Gee, and W. A. Wall. A finite deformation mortar contact formulation using a primal-dual active set strategy. International Journal for Numerical Methods in Engineering, 79(11):1354-1391, 2009.

68. A. Popp, M. Gitterle, M. W. Gee, and W. A. Wall. A dual mortar approach for 3d finite deformation contact with consistent linearization. International Journal for $\mathrm{Nu}$ merical Methods in Engineering, 83(11):1428-1465, 2010.

69. A. Prasad, N. Xiao, X.-Y. Gong, C. K. Zarins, and C. A. Figueroa. A computational framework for investigating the positional stability of aortic endografts. Biomechanics and modeling in mechanobiology, pages 1-19, 2012.

70. M. Raghavan and D. A. Vorp. Toward a biomechanical tool to evaluate rupture potential of abdominal aortic aneurysm: identification of a finite strain constitutive model and evaluation of its applicability. Journal of Biomechanics, 33(4):475 - 482, 2000.

71. M. L. Raghavan, M. M. Hanaoka, J. A. Kratzberg, M. de Lourdes Higuchi, and E. S. Da Silva. Biomechanical failure properties and microstructural content of ruptured and unruptured abdominal aortic aneurysms. Journal of biomechanics, 44(13):2501-2507, 2011.

72. D. Roy, S. Lerouge, K. Inaekyan, C. Kauffmann, R. Mongrain, and G. Soulez. Experimental validation of more realistic computer models for stent-graft repair of abdominal aortic aneurysms, including pre-load assessment. International journal for numerical methods in biomedical engineering, 32(12), 2016.

73. S. M. Sampaio, J. M. Panneton, G. I. Mozes, J. C. Andrews, T. C. Bower, M. Karla, A. A. Noel, K. J. Cherry, T. Sullivan, and P. Gloviczki. Proximal type i endoleak after endovascular abdominal aortic aneurysm repair: predictive factors. Annals of vascular surgery, 18(6):621628, 2004.

74. W. C. Sternbergh, S. R. Money, R. K. Greenberg, T. A. Chuter, Z. Investigators, et al. Influence of endograft oversizing on device migration, endoleak, aneurysm 
shrinkage, and aortic neck dilation: results from the zenith multicenter trial. Journal of vascular surgery, 39(1):20-26, 2004.

75. J. van Prehn, F. Schlösser, B. Muhs, H. Verhagen, F. Moll, and J. van Herwaarden. Oversizing of aortic stent grafts for abdominal aneurysm repair: a systematic review of the benefits and risks. European Journal of Vascular and Endovascular Surgery, 38(1):42-53, 2009.

76. J. P. Vande Geest, E. S. Di Martino, A. Bohra, M. S. Makaroun, and D. A. Vorp. A biomechanics-based rupture potential index for abdominal aortic aneurysm risk assessment. Annals of the New York Academy of Sciences, 1085(1):11-21, 2006.

77. J. P. Vande Geest, M. S. Sacks, and D. A. Vorp. The effects of aneurysm on the biaxial mechanical behavior of human abdominal aorta. Journal of biomechanics, 39(7):1324-1334, 2006.

78. J. P. Vande Geest, M. S. Sacks, and D. A. Vorp. A planar biaxial constitutive relation for the luminal layer of intra-luminal thrombus in abdominal aortic aneurysms. Journal of biomechanics, 39(13):2347-2354, 2006.

79. L. Vu-Quoc and X. Tan. Optimal solid shells for nonlinear analyses of multilayer composites. i. statics. Computer methods in applied mechanics and engineering, 192(9):975-1016, 2003.

80. D. H. Wang, M. Makaroun, M. W. Webster, and D. A. Vorp. Mechanical properties and microstructure of intraluminal thrombus from abdominal aortic aneurysm. Journal of Biomechanical Engineering, 123(6):536-539, 2001.

81. W. Wang, B. Jüttler, D. Zheng, and Y. Liu. Computation of rotation minimizing frames. ACM Transactions on Graphics (TOG), 27(1), 2008.

82. Y. G. Wolf, B. B. Hill, W. A. Lee, C. M. Corcoran, T. J. Fogarty, and C. K. Zarins. Eccentric stent graft compression: an indicator of insecure proximal fixation of aortic stent graft. Journal of vascular surgery, 33(3):481-487, 2001.

83. T. R. Wyss, F. Dick, L. C. Brown, and R. M. Greenhalgh. The influence of thrombus, calcification, angulation, and tortuosity of attachment sites on the time to the first graft-related complication after endovascular aneurysm repair. Journal of vascular surgery, 54(4):965-971, 2011. 